\title{
TANGLE AND BRAUER DIAGRAM ALGEBRAS OF TYPE $\mathrm{D}_{n}$
}

\author{
ARJEH M. COHEN \& DIÉ A.H. GIJSBERS \& DAVID B. WALES
}

\begin{abstract}
A generalization of the Kauffman tangle algebra is given for Coxeter type $\mathrm{D}_{n}$. The tangles involve a pole of order 2. The algebra is shown to be isomorphic to the Birman-Murakami-Wenzl algebra of the same type. This result extends the isomorphism between the two algebras in the classical case, which, in our set-up, occurs when the Coxeter type is $\mathrm{A}_{n-1}$. The proof involves a diagrammatic version of the Brauer algebra of type $\mathrm{D}_{n}$ of which the generalized Temperley-Lieb algebra of type $\mathrm{D}_{n}$ is a subalgebra.

KEYWORDS: associative algebra, BMW algebra, Brauer algebra, TemperleyLieb algebra, tangle, Brauer diagram, Coxeter groups

AMS 2000 Mathematics Subject Classification: 16K20, 17Bxx, 20F05, 20F36, 20M05, 57Mxx
\end{abstract}

\section{INTRODUCTION}

In 11, Morton and Wasserman described an explicit isomorphism between the Birman-Murakami-Wenzl (BMW) algebra $\mathbf{B}\left(\mathrm{A}_{n-1}\right)$ of type $\mathrm{A}_{n-1}$, which is given by means of a presentation by generators and relations, and the Kauffman tangle algebra $\mathbf{K T}\left(\mathrm{A}_{n-1}\right)$ connected to braids on $n$ strands. In this paper we introduce a tangle algebra $\mathbf{K T}\left(\mathrm{D}_{n}\right)$ with a pole of order two and show that it is isomorphic to the BMW algebra $\mathbf{B}\left(\mathrm{D}_{n}\right)$ of type $\mathrm{D}_{n}$. This construction extends the one of [11] by a pole of order two. In [1, Allcock had a similar pole involving $\mathrm{D}_{n}$. In [10], $\mathrm{R}$. Häring-Oldenburg deals with the case of type $\mathrm{B}_{n}$ but uses further relations.

We also construct a Brauer diagram algebra $\operatorname{BrD}\left(\mathrm{D}_{n}\right)$ of type $\mathrm{D}_{n}$. This algebra is constructed with a basis of diagrams, much like the diagrams Brauer used in [3]. The algebra extends Green's 9] diagrammatic description of the Temperley-Lieb algebra of type $\mathrm{D}_{n}$.

The tangle algebra $\mathbf{K T}\left(\mathrm{D}_{n}\right)$ is introduced in Definition 2.6 below. The BMW algebras $\mathbf{B}(M)$ were defined for arbitrary graphs $M$ in [5]. Here, in Definition 2.1 below, we introduce an integral version of these over the domain $R=\mathbb{Z}\left[\delta^{ \pm 1}, l^{ \pm 1}, m\right] /((1-$ $\left.\delta) m-l+l^{-1}\right)$, where $l, m, \delta$ are indeterminates. The Brauer diagram algebra $\operatorname{BrD}\left(\mathrm{D}_{n}\right)$ will be defined over the quotient $\operatorname{ring} \bar{R}=R /(l-1, m) \cong \mathbb{Z}\left[\delta^{ \pm 1}\right]$, see Definition 4.4. As in the BMW algebra case, for each graph $M$, a Brauer algebra $\operatorname{Br}(M)$ over $\bar{R}$ has been defined by generators and relations, see 4 . As described in [6, modding out $l-1$ and $m$ gives a surjective $R$-equivariant homomorphism $\mu: \mathbf{B}(M) \rightarrow \mathbf{B r}(M)$. Our main results can be summarized as follows, where $n ! !=1 \cdot 3 \cdot 5 \cdots(2 n-3)(2 n-1)$ and $d(n)=\left(2^{n}+1\right) n ! !-\left(2^{n-1}+1\right) n !$.

Theorem 1.1. The algebras mentioned have the following properties for $n \geq 2$.

(i) There is a surjective R-equivariant homomorphism $\psi: \mathbf{K T}\left(\mathrm{D}_{n}\right) \rightarrow \mathbf{B r D}\left(\mathrm{D}_{n}\right)$.

Date: October 23, 2018. 
(ii) There is a $\mathbb{Z}\left[\delta^{ \pm 1}\right]$-algebra isomorphism $\nu: \operatorname{Br}\left(\mathrm{D}_{n}\right) \rightarrow \operatorname{BrD}\left(\mathrm{D}_{n}\right)$. Both algebras are free of dimension $d(n)$.

(iii) There is an R-algebra isomorphism $\varphi: \mathbf{B}\left(\mathrm{D}_{n}\right) \rightarrow \mathbf{K T}\left(\mathrm{D}_{n}\right)$. Both algebras are free of dimension $d(n)$.

(iv) The diagram below of $R$-equivariant homomorphisms is commutative.

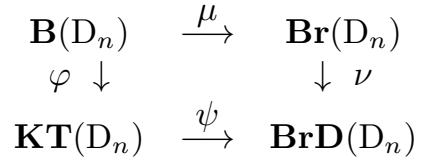

Here, the Coxeter type $\mathrm{D}_{n}$ is understood to be $\mathrm{A}_{1} \mathrm{~A}_{1}$ if $n=2$ and $\mathrm{A}_{3}$ if $n=3$.

In [8], Goodman and Hauschild gave a similar construction for affine BMW algebras and affine Kauffman tangle algebras, with a pole appearing in the tangles. Here we also define an $(n, n)$ tangle algebra, denoted $\mathbf{K T}\left(\mathrm{D}_{n}\right)^{(1)}$, with respect to a pole of order two, cf. Definition 2.6. Our tangle algebra differs from the algebras introduced in 8 in that some of our tangles with twists around the pole cannot be simplified whereas the tangles of 8$]$ can.

The work for $\mathrm{D}_{n}$ encompasses $\mathrm{A}_{n-1}$. The precise definition of the classical tangle algebra $\mathbf{K T}\left(\mathrm{A}_{n-1}\right)$ can be obtained from Definition 2.6 below after removing the pole and ignoring all relations connected to it, and so $\mathbf{K T}\left(\mathrm{A}_{n-1}\right)$ is a subalgebra of $\mathbf{K T}\left(\mathrm{D}_{n}\right)$. By restriction of $\varphi$ we find an isomorphism between the classical BMW algebra, $\mathbf{B}\left(\mathrm{A}_{n-1}\right)$, and $\mathbf{K T}\left(\mathrm{A}_{n-1}\right)$. This gives an alternative proof to the one by Morton and Wasserman in [11, cf. Remark 3.15)(ii) below.

This paper is organized as follows. In Section 2, we introduce the tangle algebras $\mathbf{K T}\left(\mathrm{D}_{n}\right)^{(1)}$ and $\mathbf{K T}\left(\mathrm{D}_{n}\right)$, recall the BMW algebra $\mathbf{B}\left(\mathrm{D}_{n}\right)$, and exhibit the homomorphism $\varphi: \mathbf{B}\left(\mathrm{D}_{n}\right) \rightarrow \mathbf{K T}\left(\mathrm{D}_{n}\right)$. The presentation by generators and relations of $\mathbf{B}\left(\mathrm{D}_{n}\right)$ gives rise to a natural homomorphism $\varphi: \mathbf{B}\left(\mathrm{D}_{n}\right) \rightarrow \mathbf{K T}\left(\mathrm{D}_{n}\right)$ of $R$-algebras. In Section 3. we introduce totally descending tangles for which Reidemeister moves can be made. We also find a standard expression for tangles in terms of closed strands and twists around the pole. This will enable us to prove that $\varphi$ is surjective, see Theorem 3.14 By [6, Theorem 1.1] the dimension of $\mathbf{K T}\left(\mathrm{D}_{n}\right)$ is at most $d(n)$. In Section 4 we deal with the Brauer diagram algebra of type $\mathrm{D}_{n}$. Knowledge from [5] helps us to identify $\operatorname{BrD}\left(\mathrm{D}_{n}\right)$ with the Brauer algebra $\operatorname{Br}\left(\mathrm{D}_{n}\right)$, see Proposition 4.8. This takes care of Theorem 1.1(ii). There is a surjective homomorphism $\psi$ of rings from the tangle algebra $\mathbf{K T}\left(\mathrm{D}_{n}\right)$ onto the Brauer diagram algebra $\operatorname{BrD}\left(\mathrm{D}_{n}\right)$, see Proposition 4.10, this establishes Theorem 1.1(i) and helps us find a lower bound for the dimension of $\mathbf{K T}\left(\mathrm{D}_{n}\right)$. These facts are used in the isomorphism proof of $\mathbf{B}\left(\mathrm{D}_{n}\right)$ and $\mathbf{K T}\left(\mathrm{D}_{n}\right)$ in Theorem 4.11, which settles Theorem 1.1 (iii) and (iv).

We finish by discussing a slightly larger tangle algebra, $\mathbf{K T}\left(\mathrm{D}_{n}\right)^{(2)}$, for which we also provide a presentation by means of generators and relations.

The work reported here grew out of the $\mathrm{Ph}$. D. thesis of one of us, 7]. The other two authors wish to acknowledge Caltech and Technische Universiteit Eindhoven for enabling mutual visits.

\section{TANgle algebras of TyPe $\mathrm{A}_{n-1}$ AND $\mathrm{D}_{n}$}

Let $M$ be a Coxeter diagram of rank $n$ without multiple bonds. We define the BMW algebra by means of $2 n$ generators and eleven kinds of relations. For each node $i$ of the diagram $M$ we define two generators $g_{i}$ and $e_{i}$ with $i=1, \ldots, n$. If 
two nodes are connected in the diagram we write $i \sim j$, with $i, j$ the indices of the two nodes, and if they are not connected we write $i \nsim j$. In this paper we will only be needing $M$ of type $\mathrm{A}_{n-1}$ and $\mathrm{D}_{n}$.

Definition 2.1. Let $M$ be a Coxeter diagram of rank $n$ without multiple bonds. The BMW algebra of type $M$ is the algebra, denoted by $\mathbf{B}(M)$, with unit element, over $R$, whose presentation is given on generators $g_{i}$ and $e_{i}(i=1, \ldots, n)$ by the following defining relations.

$\begin{array}{lllr}\text { (B1) } & g_{i} g_{j}=g_{j} g_{i} & \text { when } i \nsim j, \\ \text { (B2) } & g_{i} g_{j} g_{i}=g_{j} g_{i} g_{j} & \text { when } i \sim j, \\ \text { (D1) } & m e_{i}=l\left(g_{i}^{2}+m g_{i}-1\right) & \text { for all } & i, \\ \text { (R1) } & g_{i} e_{i}=l^{-1} e_{i} & \text { for all } i, \\ \text { (R2) } & e_{i} g_{j} e_{i}=l e_{i} & \text { when } i \sim j, \\ \text { (RSer) } & e_{i} g_{i}=l^{-1} e_{i} & \text { for all } & i, \\ \text { (HSee) } & e_{i}^{2}=\delta e_{i} & \text { for all } i, \\ \text { (HCer) } & e_{i} g_{j}=g_{j} e_{i} & \text { when } i \nsim j, \\ \text { (HCee) } & e_{i} e_{j}=e_{j} e_{i} & \text { when } i \nsim j, \\ \text { (RNrre) } & g_{j} g_{i} e_{j}=e_{i} e_{j} & \text { when } i \sim j, \\ \text { (RNerr) } & e_{i} g_{j} g_{i}=e_{i} e_{j} & \text { when } i \sim j .\end{array}$

The first two relations are the braid relations commonly associated with the Coxeter diagram $M$. Just as for Artin and Coxeter groups, if $M$ is the disjoint union of two diagrams $M_{1}$ and $M_{2}$, then $\mathbf{B}$ is the direct sum of the two BMW algebras $\mathbf{B}\left(M_{1}\right)$ and $\mathbf{B}\left(M_{2}\right)$. For the solution of many problems concerning $\mathbf{B}$, this gives an easy reduction to the case of connected diagrams $M$.

If $S$ is a ring containing $R$ in which $m$ is invertible, only the first five relations are needed as defining relations for $\mathbf{B}(M) \otimes_{R} S$; this is shown in [5. It also follows from arguments of [5] that the $g_{i}$ are invertible elements in $\mathbf{B}(M)$, so that there is a group homomorphism from the Artin group $A$ of type $M$ to the group $\mathbf{B}(M)^{\times}$ of invertible elements of $\mathbf{B}(M)$ sending the $i$-th generator $s_{i}$ of $A$ to $g_{i}$. The fact that the BMW algebras of type $\mathrm{A}_{n-1}$ coincide with those defined by Birman and Wenzl in [2] or by Murakami in [12] is given in [5, Theorem 2.7].

The other kind of algebras to be introduced are tangle algebras over $R$. We first recall from [11 the definition of a tangle as a piece of a link diagram in the plane. A $(k, n)$-tangle is a piece of a knot diagram in $\mathbb{R}^{2} \times[0,1]$, consisting of piece-wise linear curves, called strands, such that every strand intersects the boundary of $\mathbb{R}^{2} \times[0,1]$ transversally in either none or two of the points from $K=\{(1,0,1), \ldots,(k, 0,1)\} \cup$ $\{1,0,0), \ldots,(n, 0,0)\}$ and such that $K$ is the set of endpoints of strands. The elements of $K$ are called the endpoints of the tangle. A crossing of two strands is called positive if the strand moving from top right to bottom left crosses over the other strand; the opposite crossing will be called negative.

Two tangles are ambient isotopic if they are related by a sequence of Reidemeister moves I, II, and III (see Figure 1) together with isotopies of $\mathbb{R}^{2} \times[0,1]$ fixing the boundary. It is well known that the closures of two tangles represent the same knot up to isotopy if and only if they are ambient isotopic. Here we will restrict attention to regular isotopy, cf. [11.

Definition 2.2. Two tangles are said to be regularly isotopic if they are related by a sequence of only Reidemeister moves II and III together with isotopies of $\mathbb{R}^{2} \times[0,1]$ fixing the boundary. 
$\mathrm{I} / \mathrm{O}=10$
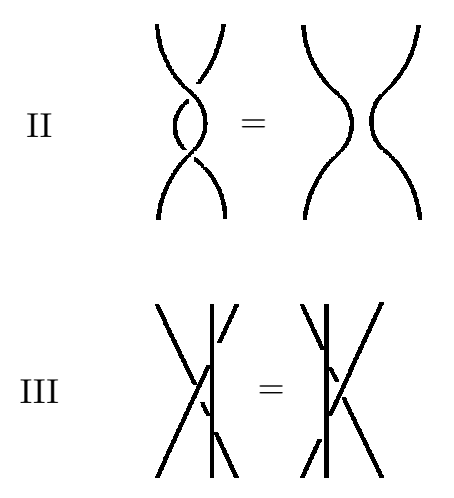

FIgURE 1. Reidemeister moves I, II, and III

A $(k, n)$-tangle and an $(n, s)$-tangle can be composed by placing the first tangle on top of the second and connecting the endpoints at the bottom of the first tangle to the endpoints at the top of the second and using an isotopy to move the set of endpoints to their standard positions.

In this paper a new set of tangles is introduced which we will call tangles of type D. These tangles have an additional strand, called a pole, with properties different from the other, ordinary strands in the tangle. We start with a general definition of a tangle with a pole. The pole will be a vertical axis, which is to the left of $K$ through $(0,0,0)$.
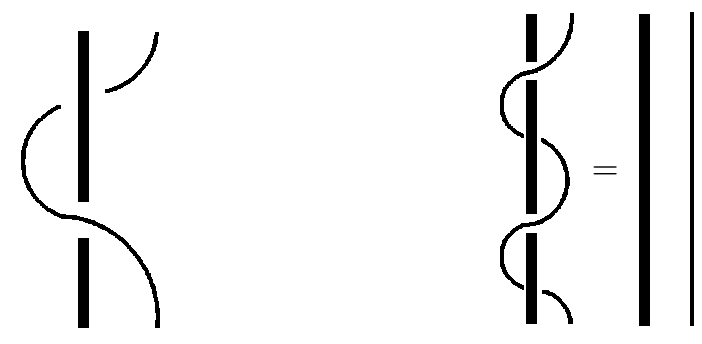

Figure 2. A pole twist and the relation of a pole of order two

Definition 2.3. An $(n, n)$-tangle with a pole is an $(n, n)$-tangle which includes the distinguished straight line segment in $\mathbb{R}^{2} \times[0,1]$ connecting $(0,0,1)$ with $(0,0,0)$, called the pole. 
While we regard normal strands of a diagram as pieces of rope or rubber bands, we can treat the pole as an iron pipe or bar. It is a fixed vertical strand which cannot be deformed (or bent). Because of this, Reidemeister I will never occur for the pole. Furthermore, we do not allow Reidemeister III where the pole is one of the three strands. Only Reidemeister II is allowed. Here two consecutive under or over crossings of one strand with the pole can be removed leaving the pole intact. It is here that we differ from [8] where Reidemeister III is allowed.

The (one time) encircling of the pole by a strand of the tangle is called a twist around the pole or simply pole twist. See Figure 2, where the pole is depicted as a bold vertical strand. For our purposes in $\mathrm{D}_{n}$ we will use a pole of order two:

Definition 2.4. The pole is said to have order two if two consecutive twists around the pole can be removed in that the resulting strand starts and finishes in the same place but no longer goes around the pole. Here consecutive means the second twist follows immediately after the first twist with no other strands between them. See Figure 2

We now define tangles of type $\mathrm{A}, \mathrm{D}^{(1)}, \mathrm{D}^{(2)}$, and $\mathrm{D}$.

Definition 2.5. An $(n, n)$-tangle of type $\mathrm{A}$ is an $(n, n)$-tangle with no strands going around the pole. An $(n, n)$-tangle of type $\mathrm{D}^{(1)}$ is an $(n, n)$-tangle with a pole of order two. If there are an even number of pole twists, it is called of type $\mathrm{D}^{(2)}$. A tangle of type $\mathrm{D}^{(2)}$ is said to be of type $\mathrm{D}$ if it has a horizontal strand whenever it has a closed strand twisting around the pole.
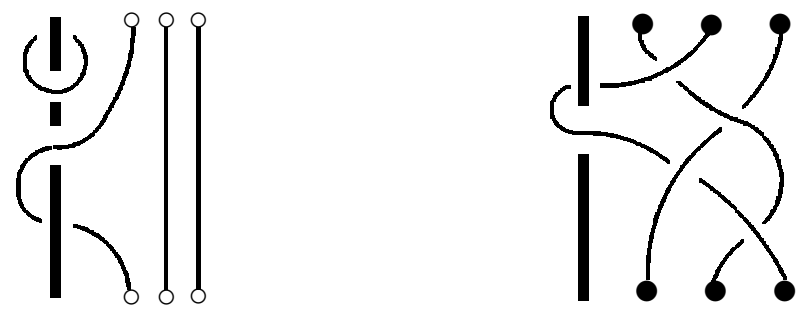

Figure 3. Two $(3,3)$-tangles, one of type $\mathrm{D}^{(2)}$ that is not of type $\mathrm{D}$, and one of type $\mathrm{D}^{(1)}$ that is not of type $\mathrm{D}^{(2)}$

As only isotopy of the plane is allowed that does not affect the pole, we forbid tangles to have crossings of strands at the left side of the pole. Moreover, all pole twists in a tangle diagram are isolated from each other. So when traversing the pole from top to bottom, the twists of a tangle are met one by one. An example of a tangle of type $\mathrm{D}^{(1)}$ is given in Figure 3

As for the Kauffman tangle algebra described in [11, we define an algebra for the tangles of type $\mathrm{D}^{(1)}$. Let $\mathcal{U}_{n}^{(1)}$ be the monoid of $(n, n)$-tangles of type $\mathrm{D}^{(1)}$ modulo regular isotopy to the right of the pole and Reidemeister II for each strand interacting with the pole. Similarly, we define the submonoids $\mathcal{U}_{n}^{(2)}$ and $\mathcal{U}_{n}$ corresponding to $(n, n)$-tangles of type $\mathrm{D}^{(2)}$ and $\mathrm{D}$, respectively. Note that the product of two tangles of each type is again of the same type. 


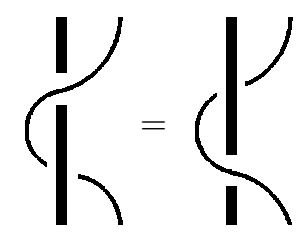

Figure 4. The double twist relation

As the tangles contain a pole of order two, the tangles in the monoid $\mathcal{U}_{n}^{(1)}$ satisfy the double twist relation, as shown in Figure 4. To see this, just compose both sides of Figure 2 with the right hand side of Figure 4 and use Reidemeister II around the pole.

Products of tangles with an even number of pole twists have an even number of pole twists, and the relations corresponding to the pole of order two preserves this. Therefore the composition of two tangles of type $\mathrm{D}^{(2)}$ is again of type $\mathrm{D}^{(2)}$.

We now introduce the tangle algebra $\mathbf{K T}\left(\mathrm{D}_{n}\right)^{(1)}$ as a quotient of the monoid algebra $R\left[\mathcal{U}_{n}^{(1)}\right]$. Later, in Definition [2.6, the algebra $\mathbf{K T}\left(\mathrm{D}_{n}\right)$ of our prime interest will appear as a subalgebra of $\mathbf{K T}\left(\mathrm{D}_{n}\right)^{(1)}$.

Definition 2.6. The tangle algebra $\mathbf{K T}\left(\mathrm{D}_{n}\right)^{(1)}$ over $R$ is the quotient algebra obtained from the monoid algebra $R\left[\mathcal{U}_{n}^{(1)}\right]$ by factoring out the following seven relations. Here, the pictures indicate tangles which differ only in the region shown.

(i) The Kauffman skein relation

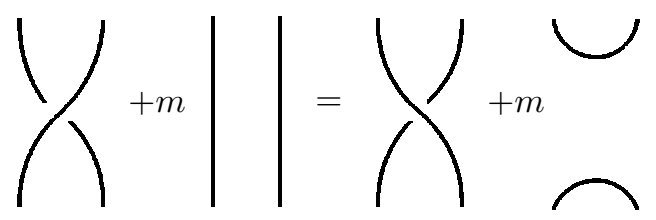

(ii) The commuting relation

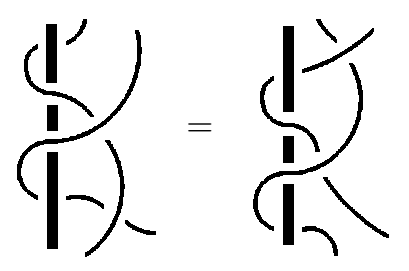

(iii) The self-intersection relations

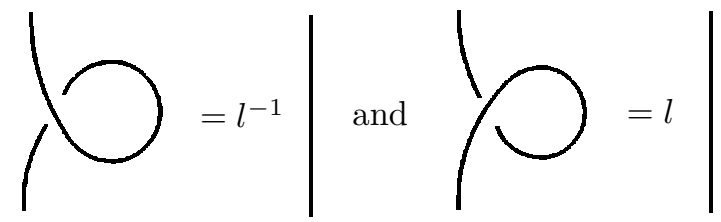

(iv) The idempotent relation

$$
T \cup O=\delta T,
$$


where $T \cup O$ is the union of a tangle $T$ and a closed loop $O$ having no crossings with $T$, no self-intersections, and no pole twists.

(v) The first pole-related self-intersection relation

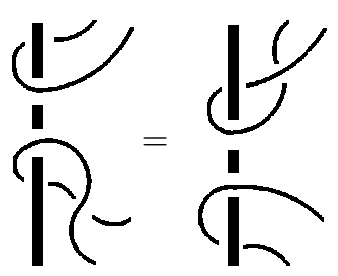

(vi) The second pole-related self-intersection relation

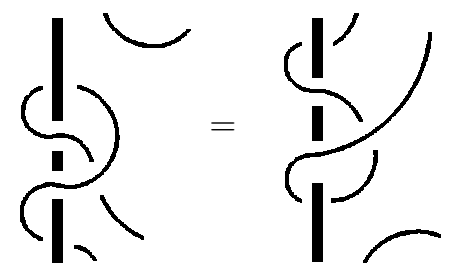

(vii) The first closed pole loop relation

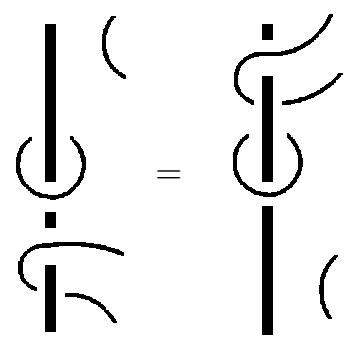

The relations generate a two-sided ideal in $R\left[\mathcal{U}_{n}^{(1)}\right]$. Thus, composition of tangles of type $\mathrm{D}^{(1)}$ induces an associative bilinear multiplication on $\mathbf{K T}\left(\mathrm{D}_{n}\right)^{(1)}$, making $\mathbf{K T}\left(\mathrm{D}_{n}\right)^{(1)}$ an algebra over $R$. The subalgebra of $\mathbf{K T}\left(\mathrm{D}_{n}\right)^{(1)}$ generated by all tangles of type $\mathrm{D}^{(2)}$ is denoted $\mathbf{K T}\left(\mathrm{D}_{n}\right)^{(2)}$.

For $n \geq 0$ define the Kauffman tangle algebra of type $\mathrm{D}$ on $n$ nodes, denoted $\mathbf{K T}\left(\mathrm{D}_{n}\right)$, to be the subalgebra of $\mathbf{K T}\left(\mathrm{D}_{n}\right)^{(2)}$ generated by all tangles of type D.

Remarks 2.7. (i). Since the relations (i)-(vii) are homogeneous with respect to the parity of the number of pole twists, $\mathbf{K T}\left(\mathrm{D}_{n}\right)^{(2)}$ is the $R$-linear span of tangles of type $\mathrm{D}^{(2)}$. Also, the Kauffman tangle algebra $\mathbf{K T}\left(\mathrm{D}_{n}\right)$ is easily seen to be the linear span of all tangles in $\mathbf{K T}\left(\mathrm{D}_{n}\right)^{(2)}$ of type $\mathrm{D}$.

(ii). In using the pole-related self-intersection relations, care must be taken to get the correct over crossings versus under crossings. In both cases, the correct diagram is obtained by turning the diagram upside down (i.e., turning the paper 180 degrees around the horizontal axis perpendicular to the pole) so that the over crossing at the bottom of the left hand side of (v) becomes an under crossing at the top of the right hand side. Recall that for pole twists without a self-intersection, there is no distinction because the pole has order two. 
(iii). If $S$ is a ring containing $R$ in which $m$ is invertible, then the relations (v), (vi), and (vii) of Definition 2.6 for $\mathbf{K T}\left(\mathrm{D}_{n}\right)^{(1)} \otimes_{R} S$ follow from the others, see [7] for details.

(iv). For $n \geq 1$, the algebras $\mathbf{K T}\left(\mathrm{D}_{n}\right)$ have the desirable property that $\mathbf{K} \mathbf{T}\left(\mathrm{D}_{n-1}\right)$ is a natural subalgebra. In fact, addition of a strand without crossings to the right side of the tangle determines a natural homomorphism $i: \mathbf{K T}\left(\mathrm{D}_{n-1}\right)^{(1)} \rightarrow \mathbf{K T}\left(\mathrm{D}_{n}\right)^{(1)}$. We also have a map $\varepsilon: \mathbf{K T}\left(\mathrm{D}_{n}\right)^{(1)} \rightarrow \mathbf{K T}\left(\mathrm{D}_{n-1}\right)^{(1)}$ defined on tangles $T$ by

$$
\varepsilon(T)=\delta^{-1} c l_{n}(T),
$$

where $c l_{n}: \mathbf{K T}\left(\mathrm{D}_{n}\right)^{(1)} \rightarrow \mathbf{K T}\left(\mathrm{D}_{n-1}\right)^{(1)}$ is the map defined by connecting the two endpoints in $K$ on the right, viz. $(n, 0,0)$ and $(n, 0,1)$, of an $(n, n)$-tangle by a strand with no crossings, self-intersections or pole twists, see Figure 5 . These maps obviously respect regular isotopy and the defining relations of $\mathbf{K T}\left(\mathrm{D}_{n}\right)^{(1)}$. As $\varepsilon \circ i(T)=T$ for $T \in \mathbf{K T}\left(\mathrm{D}_{n-1}\right)^{(1)}$, we can regard $\mathbf{K T}\left(\mathrm{D}_{n-1}\right)^{(1)}$ as a subalgebra of $\mathbf{K T}\left(\mathrm{D}_{n}\right)^{(1)}$. It is easily seen that suitable restrictions lead to embeddings of $\mathbf{K T}\left(\mathrm{D}_{n-1}\right)^{(2)}$ into $\mathbf{K T}\left(\mathrm{D}_{n}\right)^{(2)}$ and of $\mathbf{K T}\left(\mathrm{D}_{n-1}\right)$ into $\mathbf{K T}\left(\mathrm{D}_{n}\right)$.

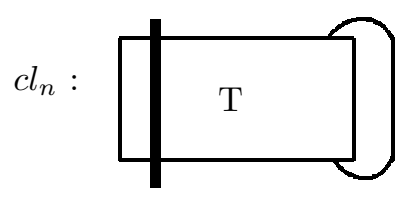

Figure 5. The closure of the rightmost strand of a tangle

We now derive a number of additional relations from the defining relations concerning small regions of the tangle diagrams containing a part of the pole. These relations will prove to be extremely useful in the full understanding of these algebras as they describe the interaction between the pole and the other strands of the tangles.

The commuting relation (ii) no longer holds if the upper crossing at each side is changed. Other variations however do hold:

Lemma 2.8. The crossings in (ii), (v), and (vi) of Definition 2.6 are all positive. These relations also hold if the signs are changed to negative as follows.

- For (ii): an upper crossing at one side and a lower crossing at the other side of the equation or all four crossings.

- For (v) and (vi): both crossings.

Proof. The first statement is evident. For (v), this follows by application of (i) to the upper crossings at both sides and identification of the terms with coefficients $m$ and $-m$ by use of (vii). For (vi), the analogous procedure works with the additional use of Reidemeister II.

For (ii), application of (i) to the upper crossing at the left hand side and the lower crossing at the right hand side and subsequent identification of the tangles with the same coefficients by means of Reidemeister II and two invocations of (v) will lead to the version of (ii) in which two crossings have changed signs. The other 
version, with left hand lower crossing and right hand upper crossing changed, is proved similarly.

For all four crossings, application of (i) to all four crossings leads to an identity of the required kind after suitable application of Reidemeister II, (vii), (vi), and the newly obtained version of $(\mathrm{v})$ to the terms that are multiples of $m$. Alternatively, two applications of the first kind gives the result.

One of the newly obtained versions of (ii) is given in Figure 6

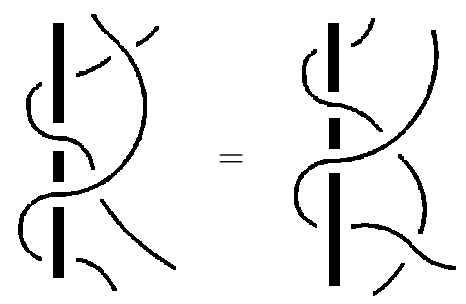

Figure 6. A second commuting relation, cf. Lemma 2.8

Relation (iii) of Definition 2.6 shows that in general a self-intersection of a strand can be replaced by $l^{ \pm 1}$. This, however, is not the case when the strand twists around the pole before intersecting itself. In contrast with the self-intersection relation, the two pole-related self-intersection relations, (v) and (vi), preserve a self-intersection albeit that the self-intersection may change strands. The strand involved twists around the pole and next crosses itself at the part of the strand before the twist. This combination of twist and self-intersection is called a polerelated self-intersection.

By the first pole-related self-intersection relation a twist of that type can be moved to neighboring twists and then to others. By the second pole-related self-intersection relation, a twist of that type can be moved to any segment of a strand which is accesible to the pole. This means there is an unobstructed region between the segment and the pole. This shows that such a pole-related self-intersection can be moved freely to many strands in the tangle. This is true even for a strand not twisting around the pole provided a segment is accessible to the pole. It can be given two pole twists by use of the double twist relation of Figure 2 (read backwards). Now the pole-related self-intersection can be moved to this strand using one of the pole-related self-intersection relations. This leads to the following observation.

Remark 2.9. If a tangle has more than one pole-related self-intersection, then by (v) and (vi) they can all be moved to a single strand. The tangle obtained in this way with more than one pole-related self-intersection can be rewritten as a linear combination of tangles with fewer pole-related self-intersections using the Kauffman skein relations. Thus each element of $\mathbf{K} \mathbf{T}\left(\mathrm{D}_{n}\right)^{(1)}$ is a linear combination of tangles with at most one pole-related self-intersection.

While working with tangles of type $\mathrm{D}$, we will encounter closed loops twisting around the pole. Here are some relations involving these loops.

Proposition 2.10. The tangles of $\mathbf{K T}\left(\mathrm{D}_{n}\right)^{(1)}$ satisfy the following relations. 
(i) The second closed pole loop relation,

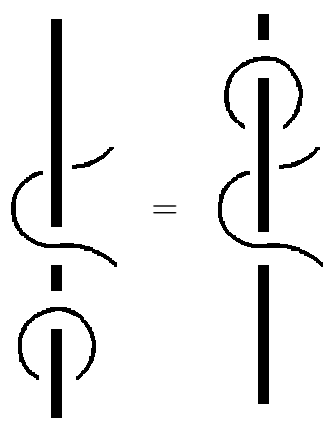

(ii) The third closed pole loop relation,

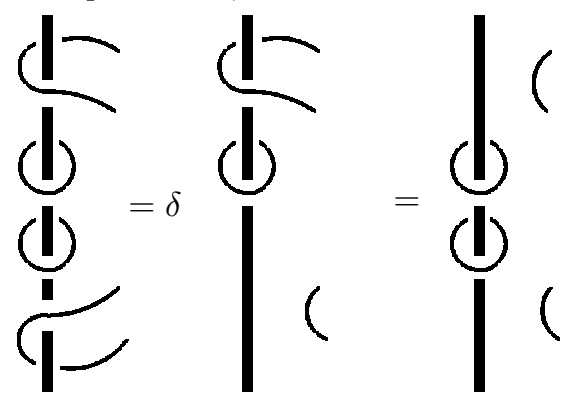

Again, the pictures indicate tangles which differ only in the region shown.

Proof. (i). Using Reidemeister II, deform the partial strand and the closed loop in such a way that, after its pole twist, the partial strand has two over crossings with the closed loop. Apply the second commuting relation, Lemma 2.8, to a large enough region containing the closed loop crossing the partial strand. Notice the over crossings become under crossings and again use Reidemeister II to shrink the loop so it does not intersect the strands. The loop is now on the other side of the partial strands. This gives (i).

(ii). This follows from the first closed pole loop relation (vii), applied to partial strands. To get the second equality, consider the bottom closed loop on the right hand diagram to be a twist around the pole joined to an arc to make it a closed loop. After applying (vii), there is an isolated closed loop not around the pole which contributes $\delta$ to the middle picture. To see that the left hand diagram is equal to the right diagram in $\mathbf{K T}\left(\mathrm{D}_{n}\right)^{(1)}$, use the second closed pole loop relation in the left hand diagram to put the bottom closed twist around the pole below the lower partial strand. Now distort the lower twist around the pole so that it has a segment curving upwards before twisting around the pole so as to be able to apply (vii). Next use (vii) to remove the twist at the top and give two twists around the pole to this lower twist. As the pole has order two, this is the diagram at the right and so they are equal in $\mathbf{K T}\left(\mathrm{D}_{n}\right)^{(1)}$.

The closed pole loop relations illustrate that when a tangle contains one closed loop around the pole, all other pole twists can be moved freely between all strands for which a segment is accessible to the pole. In particular, all these twists can be moved to a single strand. By use of the double twist relation, all but at most one 

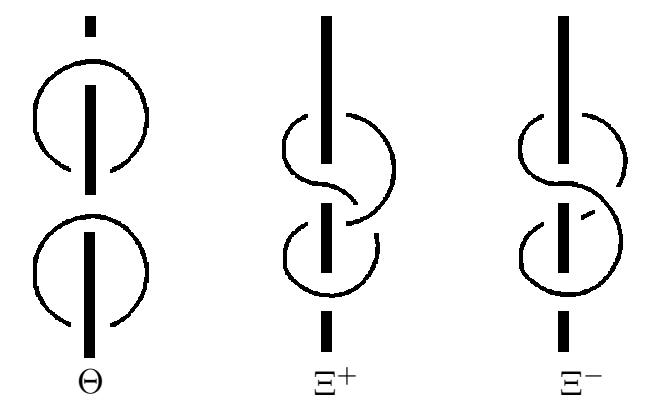

Figure 7. Three $(0,0)$-tangles of type $\mathrm{D}^{(2)}$

twist can be removed. So every tangle with closed loops and with a pole twist can be transformed to a tangle containing this closed loop with a twist around the pole and at most one other strand with a pole twist.

Furthermore, as there are an even number of twists around the pole in tangles from $\mathbf{K T}\left(\mathrm{D}_{n}\right)^{(2)}$, we can assume there are two closed loops around the pole.

We now turn our attention to certain closed strands with pole twists which we will use extensively. Denote by $\Theta$ the $(0,0)$-tangle of type $\mathrm{D}^{(2)}$ consisting of only two separate loops each of which twists around the pole, as shown in Figure 7 Denote by $\Xi^{+}$and $\Xi^{-}$the $(0,0)$-tangle of type $\mathrm{D}^{(2)}$ consisting of precisely one closed loop with two pole twists and a positive, respectively, negative self-intersection between these two twists. These $(0,0)$-tangles have some very useful properties.

Lemma 2.11. The $(0,0)$-tangles $\Theta, \Xi^{+}$, and $\Xi^{-}$satisfy the following relations in $\mathbf{K T}\left(\mathrm{D}_{0}\right)^{(2)}$.

$$
\begin{aligned}
\Xi^{+}-\Xi^{-} & =m(\Theta-\delta) \\
\left(\Xi^{+}\right)^{2} & =\delta^{2}-m \delta \Xi^{+}+m l^{-1} \delta \Theta \\
\Xi^{+} \Theta=\Theta \Xi^{+} & =\delta l^{-1} \Theta \\
\Theta^{2} & =\delta^{2} \Theta
\end{aligned}
$$

Proof. Applying the Kauffman skein relation to the single crossing in $\Xi^{+}$gives $\Xi^{+}+m \delta=\Xi^{-}+m \Theta$ and (11) follows from this, showing that $\Xi^{-}$can be expressed as a linear combination of $\Xi^{+}$and $\Theta$.

Observe that $\left(\Xi^{+}\right)^{2}$ is equal to $\delta$ times the $(0,0)$-tangle containing one closed loop around the pole with two pole-related self-intersections, as shown in Figure 8 . Property (2) is a direct result of applying the Kauffman skein relation to one of the two pole-related self-intersections.

Now (3) follows from applying the first pole-related sef-intersection relation to $\Xi^{+} \Theta$, moving the pole-related self-crossing to the nearest loop.

Also, (4) is obtained by use of the third pole-related self-intesection relations to move two twists to the same loop, resulting in two loops without pole twists to give $\delta^{2} \Theta$. 


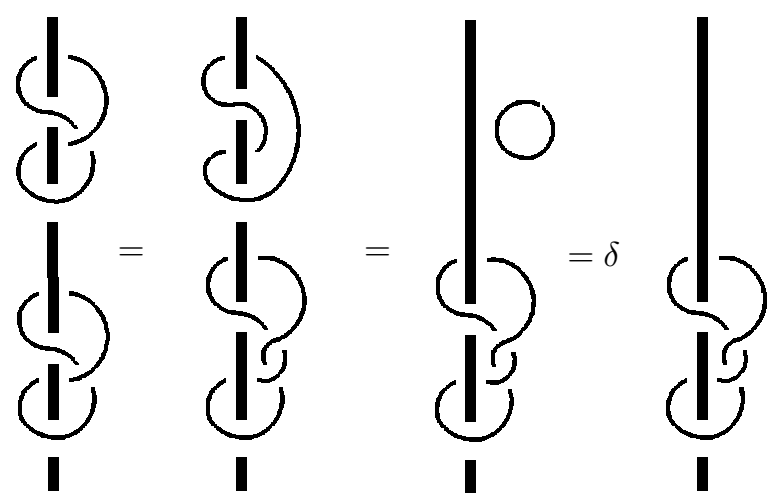

Figure 8. Equating $\left(\Xi^{+}\right)^{2}$ to $\delta$ times a closed loop with two self-intersections

The tangle $\Xi^{-}$is not the inverse of $\Xi^{+}$. However, the first pole-related selfintersection relation can be used to verify that the tangle $\delta^{-2} \Xi^{-}$is the inverse of $\Xi^{+}$.

Lemma 2.12. The tangles $\Theta, \Xi^{+}$commute with every tangle of type $\mathrm{D}^{(1)}$. In particular, $\mathbf{K T}\left(\mathrm{D}_{n}\right)^{(1)}$ can be viewed as an algebra over $R\left[\Theta, \Xi^{+}\right]$.

Proof. Both tangles obviously commute with any tangle of type $\mathrm{D}^{(1)}$ which contains no pole twists. If we can show that both tangles commute with every twist around the pole we are done.

For a closed loop with a twist around the pole this holds by Proposition 2.10 (i), the second closed pole loop relation. Hence, $\Theta$ commutes with every tangle of type $\mathrm{D}^{(1)}$.

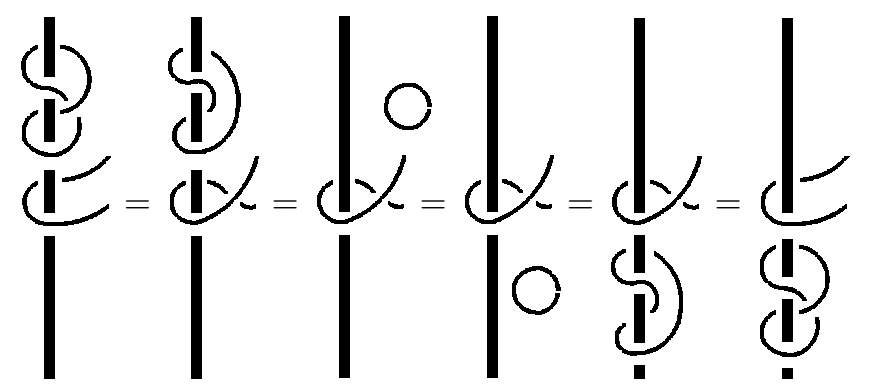

Figure 9. Commuting $\Xi^{+}$with another twist around the pole

It remains to prove that $\Xi^{+}$commutes with every pole twist. We illustrate the argument in Figure 9. Using the first pole-related self-intersection relation, we move the pole-related self-intersection of $\Xi^{+}$to the other strands twisting around the pole. The two pole twists of the closed loop without self-intersection can now be removed as the pole has order two. As a closed loop can move freely through the tangle by Reidemeister move II, we can move it to the other side of the twist. Finally, apply the reverse of the procedure just described to bring the pole-related self-intersection back in the closed loop. 
The importance of the lemma is to allow us to isolate closed strands from the tangle by commuting any $\Xi^{ \pm}$or $\Theta$ away from other parts of the tangle.

Also $\mathbf{K T}\left(\mathrm{D}_{n}\right)^{(2)}$ is an algebra over $R\left[\Xi^{+}, \Theta\right]$. But $\mathbf{K T}\left(\mathrm{D}_{n}\right)$ is not, as the multiple of the identity element by $\Xi^{+}$does not belong to it (see Definitions 2.5 and 2.6).

Our goal is to establish that $\mathbf{K T}\left(\mathrm{D}_{n}\right)$ is the image of an algebra homomorphism $\varphi: \mathbf{B}\left(\mathrm{D}_{n}\right) \rightarrow \mathbf{K T}\left(\mathrm{D}_{n}\right)^{(2)}$ for $n \geq 2$. To set up the homomorphism $\varphi$ the generators of $\mathbf{B}\left(\mathrm{D}_{n}\right)$ are to be mapped onto simple tangles which contain at most one crossing. We introduce $2 n$ of these simple tangles, which we denote by $G_{i}$ and $E_{i}$ for $i=$ $1, \ldots, n$.

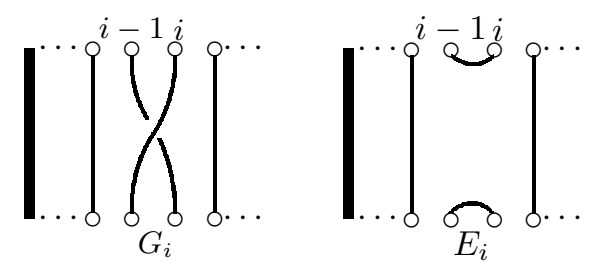

Figure 10. The tangles $G_{i}$ and $E_{i}$ for $i=2, \ldots, n$

For $i \neq 1$ we define the $G_{i}$ to be just the simple tangles where the $(i-1)$-st and $i$-th node are connected by two strands with a positive crossing. All other nodes are connected by straight lines without crossings. These tangles do not have any pole twists, see the left side of Figure 10.

The tangle $E_{i}$, where $1<i \leq n$, connects the $(i-1)$-st and $i$-th node by horizontal strands. All other nodes are again connected by straight lines without crossings. These tangles have no pole twists, see the right side Figure 10

The two tangles $G_{1}$ and $E_{1}$ are tangles with pole twists. The tangle $G_{1}$ is obtained from $G_{2}$ in a natural way by twisting the two strands connecting the first and second node around the pole. Similarly, the tangle $E_{1}$ is obtained from $E_{2}$ by twisting the two strands connecting the first and second node around the pole, see Figure 11
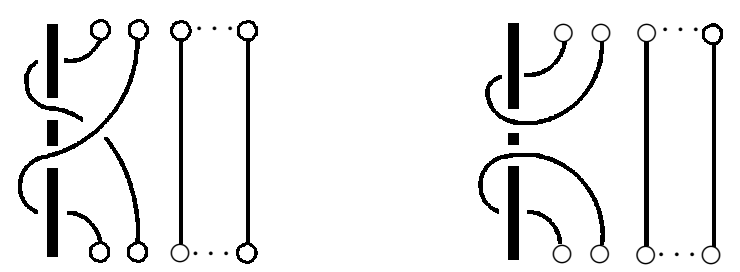

Figure 11 . The additional generators $G_{1}$ and $E_{1}$

Remarks 2.13. (i). The $G_{i}$ and $E_{i}$ for $i \geq 2$ generate a subalgebra of $\mathbf{K T}\left(\mathrm{D}_{n}\right)$ that is isomorphic with $\mathbf{K T}\left(\mathrm{A}_{n}\right)$, the Kauffman Tangle algebra as defined by [11. The isomorphism is simply defined by removing or adding the pole, which does not affect the tangles in the subalgebra generated by $G_{i}$ and $E_{i}$ for $i \geq 2$.

(ii). Define $G_{0}$ to be the tangle in Figure 12, It is of type $\mathrm{D}^{(1)}$ but not of type D. 


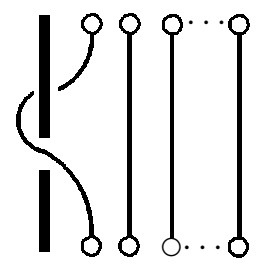

Figure 12. The element $G_{0}$

As the pole has order two, it is an involution. Conjugation by $G_{0}$ is an automorphism of $\mathbf{K T}\left(\mathrm{D}_{n}\right)^{(1)}$ as it leaves invariant the set of defining relations for $\mathbf{K T}\left(\mathrm{D}_{n}\right)^{(1)}$; for instance, it interchanges the first two pole-related self-intersection relations (v) and (vi). It also leaves the subalgebras $\mathbf{K T}\left(\mathrm{D}_{n}\right)^{(2)}$ and $\mathbf{K T}\left(\mathrm{D}_{n}\right)$ invariant. Moreover, $G_{1}=G_{0} G_{2} G_{0}$, and $E_{1}=G_{0} E_{2} G_{0}$, whereas $G_{0} G_{i} G_{0}=G_{i}$ for $i \geq 3$, so conjugation by $G_{0}$ is also an automorphism of the subalgebra of $\mathbf{K T}\left(\mathrm{D}_{n}\right)^{(1)}$ generated by the $G_{i}$ and $E_{i}$ for $1 \leq i \leq n$.

Proposition 2.14. If $n \geq 2$, there is a homomorphism $\varphi: \mathbf{B}\left(\mathrm{D}_{n}\right) \rightarrow \mathbf{K T}\left(\mathrm{D}_{n}\right)$ of $R$-algebras determined by $\varphi\left(g_{i}\right)=G_{i}$ and $\varphi\left(e_{i}\right)=E_{i}$ for all $i \in\{1, \ldots, n\}$.

Proof. We need to check that the defining relations of the BMW algebra $\mathbf{B}\left(\mathrm{D}_{n}\right)$, given in Definition 2.1 are respected by the tangles. If the indices of the generator symbols do not include 1 , the pole is not involved and the equalities follow from 11. Also, the defining relation (D1) is covered by the Kauffman skein relation (i). If one of the indices is 1 and the second not 2 , conjugate by $G_{0}$ to get $E_{2}$ or $G_{2}$. The relations can be obtained this way except for ones involving both $i=1$ and $j=2$. The relation (B1), $G_{1} G_{2}=G_{2} G_{1}$, is straightforward from the commuting relation (i). The relation (HCer), $E_{1} G_{2}=G_{2} E_{1}$, follows from the first pole-related self-intersection and conjugation by $G_{0}$. The relation $E_{2} G_{1}=G_{1} E_{2}$ follows by conjugation with $G_{0}$. Finally, the relation (HCee), $E_{1} E_{2}=E_{2} E_{1}$, follows from the first closed pole loop relation and the pole being of order two.

Later, in Theorem 3.14 we will show that $\varphi$ is surjective.

\section{Totally Descending Tangles}

In this section we identify a spanning set of tangles for $\mathbf{K T}\left(\mathrm{D}_{n}\right)$. The result is Proposition 3.13 below. We will restrict our attention to certain tangles, called totally descending, as in [11].

Definition 3.1. Given a tangle $T$, choose a sequence of base points: firstly an endpoint (in $K$ ) for each full non-closed strand, and, secondly a point on each closed strand. Subsequently provide each strand with an orientation determined by a direction starting at the base point. We say $T$ is totally descending (with respect to the ordered base points and orientations) if, on traversing all the strands from $T$ in order of the base points, we meet each crossing for the first time as an over crossing. Such a crossing is called descending.

Lemma 3.2. $\mathbf{K T}\left(\mathrm{D}_{n}\right)$ is spanned by totally descending tangles. 
Proof. The proof is similar to the proof of Theorem 2.6 in 11 and is done by induction on first the number of crossings, then on the number of non-descending crossings.

Let $T$ be a tangle in $\mathbf{K T}\left(\mathrm{D}_{n}\right)$. Choose a sequence of base points for $T$. Follow the strands of $T$ in this determined order. At the first non-descending crossing apply the Kauffman skein relation (i). This results in a linear combination of a tangle with this particular crossing changed to a descending one and two tangles with the crossing removed. Induction shows we can write every tangle in $\mathbf{K T}\left(\mathrm{D}_{n}\right)$ as a linear combination of totally descending crossings this way.

Each strand of a totally descending tangle lies entirely above the strands that appear later in the order. The importance of totally descending tangles is that Reidemeister moves can always be made. In the case of Reidemeister III the move is with respect to at least one of the crossings. This is because one of the strands is first in the order given by Definition 3.1 and one is last. This means one is above the other two, and one below the other two and stays that way. For Reidemeister II one is above the other.

The next result involves eight kinds of regions always to the right of the pole.

Definition 3.3. Let $T$ be a tangle in $\mathcal{U}_{n}^{(1)}$. A region of $T$ is understood to be a part of the $x, z$-plane $\Pi$ in which $T$ is projected by means of the natural projection along the $y$-axis; it is bounded by segments of strands and segments from the borders of the diagram, with the understanding that the West border of $T$ consists of the pole; so parts of the strands that are left of the pole never occur as parts of a region. In particular, a region can be crossed by strands. Crossings of strands will be interpreted as real crossings in the plane $\Pi$ where the boundaries of regions are concerned, even though in $T$ one strand of the crossing passes above the other. A region of $T$ is called of type $\mathrm{E}$ if one of the following cases occurs.

(1) The region is bounded by exactly three strands as in Reidemeister III.

(2) The region is bounded by exactly two strands as in Reidemeister II or by just one self-intersecting strand as in Reidemeister I; or the region is enclosed by a strand twisting around the pole exactly once with one selfintersection as in a pole-related self-intersection, in which case the region is bounded by the part of the pole where the strand twists around the pole and the segments of the strand until they cross to the right of the pole.

(3) The region is bounded by the East border of $T$ and a single strand, which starts at $n$ on the top (that is, endpoint $(n, 0,1))$ and finishes at $n$ on the bottom (endpoint $(n, 0,0)$ ).

(4) The region is bounded by the segment of the North border between endpoints $i$ and $i+1$, and by a single strand starting at $i$ on the top and ending at $i+1$ on the top or by the two strands starting at the top endpoints $i$ and $i+1$, respectively. Similarly, the same description using the South border.

(5) The region is bounded by a segment of the pole and the segment of a strand between two pole twists if no other strand twists around the pole between these two twists.

(6) The region is bounded by the topmost segment of the pole, the leftmost segment at the top between the pole and the strand starting at the endpoint 1 , and the segment of the strand starting at top endpoint 1 and its first 
twist around the pole if no other strand has twisted around the pole in this region.

(7) The region is enclosed by a closed strand without self-intersections. The strand is either entirely to the right of the pole or twists around the pole exactly once, in which case the region is bounded by the part of the pole where the strand twists around the pole and the segment of the strand to the right of the pole.

(8) The region is bounded by the pole and by two strands each of which twists around the pole while the two strands cross to the right of the pole without any pole twists in between.

Lemma 3.4. Suppose that $Q$ is a region of type $E$ of a totally descending tangle in $\mathcal{U}_{n}^{(1)}$ without closed strands. Then $Q$ can be evacuated in the sense that all strands entering the region can be removed by means of Reidemeister moves. The resulting tangle is totally descending, represents the same element of $\mathbf{K T}\left(\mathrm{D}_{n}\right)^{(1)}$, and has no strands in the interior of $Q$.

Proof. We begin with $Q$ being a region of type (1) or (2). We will use induction on the total number of crossings in $Q$, including those in its boundary. If $Q$ is not already evacuated, there are strands which enter $Q$ and subsequently leave it. Let $s$ be such a strand. By induction, we can evacuate any region bordered by a self-intersection of $s$ within $Q$ and subsequently apply Reidemeister I. Therefore, we may assume that $s$ has no self-intersections within $Q$. If it enters and leaves across the same strand segment of the boundary of $Q$, it creates a new region of type (2) and within this region there are fewer total crossings than $Q$ has and, by the induction hypothesis we can evacuate this region; using Reidemeister II we can take $s$ completely away from $Q$ and use induction again. Assume therefore that $s$ leaves through a different strand in the boundary of $Q$. If $Q$ is of type (2), there are two new regions of type (1). Each has fewer total crossings as at least one of the original crossings is not in the new region. Use induction again to evacuate one of the new regions. If $Q$ is of type (1), at least one of the two new regions of the dissection of $Q$ by $s$ is of type (1). Again the new regions have fewer total crossings and so, by induction, can be evacuated. In both cases, we use of Reidemeister III to remove $s$ from $Q$. This shows that the result holds for regions of type (1) and type (2).

Now suppose that $Q$ is a region of another type. If a strand enters $Q$, it must also leave it. By following a strand from when it enters $Q$ until it first leaves $Q$, we obtain a new region of type (1) or type (2). If there is just one strand bordering the region it is certainly of type (2). When there are two strands it could be of type (1). Now use the result above for regions of these types to remove the strand from $Q$. Continue doing this until all extraneous strands are removed from $Q$.

Proposition 3.5. Suppose that $T$ is a totally descending tangle in $\mathcal{U}_{n}^{(1)}$ and let $Q$ be a region of type $E$. Then $Q$ can be evacuated in the sense that the tangle can be rewritten to a tangle that is totally descending, represents the same element of $\mathbf{K T}\left(\mathrm{D}_{n}\right)^{(1)}$, and has no strands in the interior of $Q$. Moreover, every closed strand to the right of the pole can be removed with the introduction of powers of $\delta$ and $l^{ \pm 1}$ as coefficients of $T$ in $\mathbf{K T}\left(\mathrm{D}_{n}\right)^{(1)}$.

Proof. By the definition of type E, there are no closed strands twisting around the pole inside $Q$. If there are no closed strands inside $Q$, then by Lemma 3.4 we can 
evacuate $Q$ as required. So assume $q$ is a closed strand inside $Q$, not enclosing another closed strand. The region enclosed by $q$ is made up of a finite number of regions entirely bounded by a single segment of $q$. Such a region is called a closed component. Notice that each of the closed components can be evacuated by Lemma 3.4 and removed by Reidemeister I with the introduction of a power of $l$ until there is just one closed component which can also be evacuated. Such a closed component can be shrunk to a very small one which can be moved by Reidemeister II across any strand and can be completely separated from the rest of the diagram. It can then be removed by multiplying by $\delta$ using the idempotent relation (iv).

In view of Proposition 3.5, we can evacuate certain regions of a tangle in $\mathcal{U}_{n}^{(1)}$ while representing the same element of $\mathbf{K T}\left(\mathrm{D}_{n}\right)^{(1)}$. In order to keep track of the form of tangles needed for a spanning set of $\mathbf{K T}\left(\mathrm{D}_{n}\right)$, we introduce the following two notions.

Definition 3.6. The complexity of a tangle $T$ in $\mathcal{U}_{n}^{(1)}$ is the sum of the number of pole twists and the number of crossings appearing in $T$. Such a tangle is called reduced if, as a member of $\mathbf{K T}\left(\mathrm{D}_{n}\right)^{(1)}$, it is totally descending and cannot be written as a linear combination of tangles with strictly lower complexities.

In our search for a spanning set of $\mathbf{K T}\left(\mathrm{D}_{n}\right)$ we need only consider reduced tangles. In a reduced tangle, no self-intersecting strands without pole twists occur; for otherwise a region of type E(2) would occur, which can be evacuated by Proposition 3.5. so that Reidemeister I could be applied to reduce the tangle's complexity. Similarly, by Reidemeister II, no two strands cross twice without twisting around the pole between the two crossings. Similarly, up to scaling by factors of $\delta$, we can assume that no closed strands occur to the right of the pole. As discussed in Remark 2.9, a reduced tangle has at most one pole-related self-intersection.

Lemma 3.7. Let $T$ be a tangle in $\mathcal{U}_{n}^{(1)}$ with a strand $q$ having two pole twists without pole-related self-intersections. Assume that there are no further twists of $q$ around the pole in between these two. Let $Q$ be the region bounded by the pole between the two twists and the segment of $q$ between the two twists. If $Q$ has no closed strands, then $T$ is not reduced.

Proof. Assume that $T$ is a counterexample of smallest complexity. Let $t_{1}$ and $t_{2}$ be the two consecutive twists of $q$ around the pole and bordering $Q$. If there are no strands of $T$ inside $Q$, the twists $t_{1}$ and $t_{2}$ are adjacent along the pole and, as the pole has order 2 , these twists can be removed. As $T$ is reduced any strand entering $Q$ must twist around the pole before leaving $Q$. So without loss of generality we may assume there is a further pole twist, say $t_{3}$ of a strand $q^{\prime}$ between $t_{1}$ and $t_{2}$. Since, by assumption, there are no closed strands within $Q$, the strand $q^{\prime}$ enters and leaves $Q$. If $q^{\prime}$ twists around the pole twice, there is a smaller region as in the hypotheses, and the result follows from the minimality of the complexity of $T$. If one of the twists of $q^{\prime}$ were a pole-related self-intersection within $Q$, it could be moved to $q$ at $t_{1}$ or $t_{2}$ by Lemma 2.8. Therefore, we may assume that each strand entering $Q$ twists once inside the region.

Without loss of generality, we may assume that $t_{3}$ is the first twist occurring in between $t_{1}$ and $t_{2}$ when going down the pole. This implies that the region bounded by the segment of the pole from $t_{1}$ to $t_{3}$ and by $q$ and $q^{\prime}$ has type $\mathrm{E}(8)$ and can be evacuated as in Lemma 3.4. Let $Q^{\prime}$ be the region whose corners are the twist 
$t_{3}$ and the two adjacent crossings of $q$ and $q^{\prime}$. If $Q^{\prime}$ is also evacuated, the second commuting relation allows us to remove the twist from $Q$, a contradiction.

We are left with the case where $Q^{\prime}$ is not evacuated. So there is a strand $q^{\prime \prime}$ that enters $Q^{\prime}$ and a region $S$ of type E(1) whose corners are a crossing of $q$ and $q^{\prime}$, a crossing of $q$ and $q^{\prime \prime}$, and a crossing of $q^{\prime}$ and $q^{\prime \prime}$. Evacuate $S$ and apply Reidemeister III so as to remove $q^{\prime \prime}$ from $Q^{\prime}$. By induction on the number of crossings in $Q^{\prime}$, we can evacuate all of $Q^{\prime}$ in this way. This leads us to the previous case and hence to the final contradiction.

Lemma 3.8. Let $T$ be a tangle in $\mathcal{U}_{n}^{(1)}$ for which there are no closed strands which twist around the pole. Suppose that some strand of $T$ twists around the pole two or more times without a pole-related self-intersection. Then $T$ is not reduced.

Proof. Suppose that $T$ is reduced. If there is a strand in $T$ without a pole-related self-intersection that twists around the pole more than once, by the assumption that there are no closed strands, Lemma 3.7 allows us to rewrite $T$ to a linear combination of tangles of smaller complexities.

We next deal with closed strands which twist around the pole. Examples are the closed tangles $\Theta, \Xi^{+}$, and $\Xi^{-}$which occurred in Lemma 2.11 and Figure 7 . When there are no other strands crossing such a closed strand, it can be moved into the coefficient ring $R\left[\Xi^{+}, \Theta\right]$, cf. Lemma 2.12. We will show that when there is such a closed strand, the tangle can be rewritten so that the strand has at most two twists around the pole.

Lemma 3.9. Suppose that $T$ is a tangle in $\mathcal{U}_{n}^{(1)}$ containing a closed strand $q$ twisting around the pole three or more times. Assume there is no other closed strand twisting around the pole between any two of these twists. Then $T$ is not reduced.

Proof. Let $T$ be a counterexample of minimal complexity. By Remark 2.9, of all twists around the pole in $T$, at most one is a pole-related self-intersection, and this one can be moved to the South-most twist, say $t_{3}$, of $q$ around the pole. Consider the top twist $t_{1}$ of $q$ around the pole. The two segments of $q$ beginning at $t_{1}$ do not cross as these segments could not possibly both end at $t_{3}$ for otherwise they would close the strand and $q$ would have at most two twists. Let $t_{2}$ be the next twist of $q$ from the top. The bottom segment of $q$ starting at $t_{1}$ must be joined to the top segment of $q$ starting at $t_{2}$, for otherwise, we would be able to produce another pole-related self-intersection by evacuation of a region of type E (cf. Proposition 3.5). Now the region bounded by this segment of $q$ and the part of the pole between $t_{1}$ and $t_{2}$ satisfies the conditions of Lemma 3.7. By that lemma, $T$ is not reduced, a contradiction.

Lemma 3.10. Suppose that $T$ is a reduced tangle of type $\mathrm{D}^{(1)}$ containing a closed strand $q$ that twists around the pole. If $q$ twists around the pole more than once, assume that there are two twists such that no closed strand twists around the pole between them. Then $T$ can be rewritten in such a way that $q$ is one of $\Theta, \Xi^{+}$, or $\Xi^{-}$, and no other strands cross it.

Proof. If $q$ has only one twist, the strand is a closed circle around the pole and so, by Proposition 2.10(ii) and the fact there are an even number of pole twists, we can replace $q$ by $\Theta$ up to multiplication by a power of $\delta$. 
Therefore we assume that there are no closed strands with at most one twist around the pole. In particular, $q$ has at least two pole twists, say $t_{1}$ and $t_{2}$. As the number of closed strands is finite, without loss of generality, we may assume that there is no closed strand entirely contained in the region enclosed by $q$ and the part of the pole between $t_{1}$ and $t_{2}$. Now, by restricting to a suitable region entirely containing $q$ and isotopy, we find a tangle satisfying the conditions of Lemma 3.9 Applying the lemma and continuing this way, we obtain the required assertion.

Lemma 3.11. Suppose that $T$ is a reduced tangle in $\mathcal{U}_{n}^{(2)}$. Then $T$, viewed as an element of $\mathbf{K T}\left(\mathrm{D}_{n}\right)^{(2)}$, is an $R\left[\Theta, \Xi^{+}\right]$-linear combination of tangles without closed strands. In particular, any (0,0)-tangle can be written in terms of the R-algebra $R\left[\Theta, \Xi^{+}\right]$.

Proof. Recall that $\Xi^{ \pm}$and $\Theta$ can be commuted out of $T$ by Lemma 2.12 .

Let $q$ be a closed strand. If it has no pole twists, it can be replaced by the scalar $\delta$. If $q$ has a single pole twist, it can be commuted out by the second closed pole loop relation (i) of Proposition 2.10 As the number of pole twists is even, there must be another pole twist, which we use to apply the third closed pole loop relation, Proposition 2.10(ii), and to extract a factor $\delta^{-1} \Theta$.

Suppose, therefore, that $q$ has at least two pole twists. By Lemma 3.10, we may assume that if $t_{1}$ and $t_{2}$ are a consecutive pair of pole twists, there is a closed strand twisting around the pole between them, which we can rewrite as required by induction on the number of pole twists of other strands in the region enclosed by $q$.

We have developed enough properties for a standard expression in terms of closed strands and twists around the pole.

Proposition 3.12. Let $T$ be a reduced tangle in $\mathbf{K T}\left(\mathrm{D}_{n}\right)^{(2)}$.

(i) If $T$ contains a pole-related self-intersection, then $T=\delta^{-1} \Xi^{ \pm} T^{\prime}$, where $T^{\prime}$ is the tangle obtained from $T$ by removing the pole-related self-intersection.

(ii) If $T$ contains a closed strand $q$ without self-intersections twisting around the pole, then $T=\delta^{-1} \Theta T^{\prime}$ where $T^{\prime}$ is the tangle obtained from $T$ by removing $q$ and all twists of other strands around the pole.

Proof. (i). By assumption, part of the tangle $T$ is similar to one of the partial diagrams shown in the first or second pole-related self-intersection relations (v), (vi) of Definition 2.6.

For the second pole-related self-intersection relation (vi), consider Figure 13 A closed loop with no pole twists can be brought into the tangle by applying the idempotent relation (iv) backwards. Now the self-intersection relations (iii) allow moving the pole-related self-intersection inside this loop, obtaining the tangle $T^{\prime}$ as described above.

For the first pole-related self-intersection relation (v), take the closed loop not twisting around the pole and let it twist around the pole twice and a similar picture gives the result.

(ii). Besides the single pole twist of $q$, there are an odd number, say $2 r+1$, of pole twists in $T$. As in the proof of Lemma 3.11, the second equality of the third closed pole relation, Proposition 2.10(ii), allows us to replace one of the $2 r+1$ twists by $\delta^{-1}$ and a second loop around the pole. Now the further equalities of the third 
closed pole relation can be used to remove the $2 r$ remaining pole twists and we obtain the desired tangle.
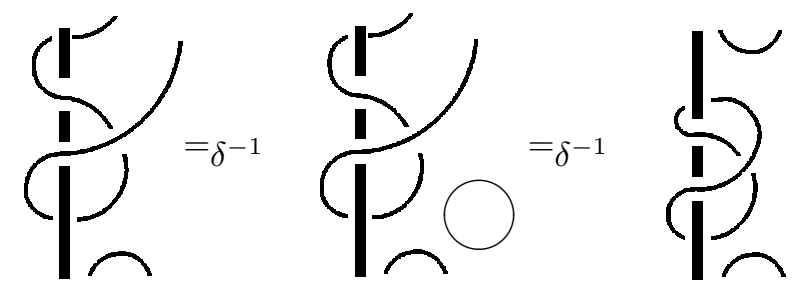

FIgURE 13. Replacing a pole-related self-intersection by $\delta^{-1} \Xi^{+}$

Theorem 3.13. As an $R$-module, $\mathbf{K T}\left(\mathrm{D}_{n}\right)$ is spanned by reduced tangles $T$ with each strand twisting around the pole at most once satisfying one of the following three properties.

(i) The tangle $T$ contains no closed strands at all.

(ii) $T=\Xi^{ \pm} T^{\prime}$ where $T^{\prime}$ is a tangle with a horizontal strand but no closed strand.

(iii) $T=\Theta T^{\prime}$ where $T^{\prime}$ is a tangle with a horizontal strand containing neither a closed strand nor a strand twisting around the pole.

Proof. By induction on complexity, there is a spanning set consisting of reduced tangles. Let $T$ be a member of a minimal spanning set of reduced tangles. If there are no crossings or twists, the tangle is of the required form. The three distinct shapes (i), (ii), (iii) are due to the rewrite rules of Proposition 3.12 and the requirement that the tangle be of type $\mathrm{D}$.

Without loss of generality we can assume that the base point and orientation on $\Xi^{+}$are chosen in such a way that the positive self-intersection is totally descending. Other configurations of closed loops around the pole are already shown to be rewritable to the ones listed here. So we only have to show that all these tangles have no closed strands without pole twists. But such closed strands can be removed as we saw in Proposition 3.5

We are now ready for one of the main results which is half of Theorem 1.1 (iii).

Theorem 3.14. The map $\varphi: \mathbf{B}\left(\mathrm{D}_{n}\right) \rightarrow \mathbf{K T}\left(\mathrm{D}_{n}\right)$ of Proposition 2.14 is surjective.

Proof. Recall that $n \geq 2$. We need to show that an arbitrary reduced tangle is a monomial in the generators $G_{i}^{ \pm 1}, E_{i}$ for $i=1, \ldots, n$.

Suppose that the result is proved for reduced tangles without closed strands and let $T$ be as in (ii) or (iii) Theorem 3.13 Then the tangle $T^{\prime}$ in this part of the theorem is in the image of $\varphi$. We can conjugate such a $T^{\prime}$ by a suitable series of $G_{i}$ 's so a horizontal strand joins the endpoints 1 to 2 at the top. By Remark 2.13(ii), conjugation by $G_{0}$ is an isomorphism of the algebra generated by these generators, so we can conjugate by $G_{0}$ if necessary to reduce to the case where the strand does not go around the pole (here we use the fact, given by the theorem, that each strand of $T$ twists around the pole at most once).

Now premultipy by $E_{2} G_{1} E_{2}$ or $E_{2} E_{1} E_{2}$ to get the factor $\Xi^{ \pm}$or $\Theta$ and so $T$ is also in the image of $\varphi$. 
Therefore, by Theorem 3.13, we may assume that $T$ has no closed strands. Suppose first that there is a vertical strand going from the top to the bottom. If it happens to twist around the pole, it goes around the pole exactly once, by Theorem 3.13 Now multiply on the left and the right by products of $G_{i}$, with $i \geq 2$ to get that 1 on the top goes to 2 on the bottom and of course still around the pole. Now conjugate by $G_{0}$ to get a vertical strand from 1 on the top to 2 on the bottom that does not twist around the pole.

Suppose then that there is a vertical strand that does not twist around the pole. Now multiply again on the left and the right by products of $G_{i}$, with $i \geq 2$ to get that $n$ on the top goes to $n$ on the bottom. Now this strand forms a region of type $\mathrm{E}(3)$, cf. Definition 3.3. and so can be evacuated by Lemma 3.4. The result is an $(n-1, n-1)$-tangle $T_{1}$ with $T=\varepsilon\left(T_{1}\right)$ and we can use induction unless $n=2$. Suppose $n=2$. As there are an even number of twists around the pole we see the other strand must join 1 on the top to 1 on the bottom without twisting around the pole. As there are no horizontal strands and the element is in $\mathbf{K T}\left(\mathrm{D}_{n}\right)$ there are no closed loops twisting around the pole and so $T$ is the identity, and so belongs to the image of $\varphi$.

The only other possibility is that all strands are horizontal. Suppose that there are horizontal strands on the top and on the bottom that do not go around the pole. Act by $G_{i}, i \geq 2$ to get strands from the endpoint $n-1$ on the bottom to $n$ on the bottom and the same for the top. Now these strands bound regions of type $\mathrm{E}(4)$ and can be evacuated by Lemma 3.4. This leaves an $(n-2, n-2)$-tangle $T_{2}$ with $T=T_{2} E_{n}$, and we use induction on $n$ for $T_{2}$ to conclude that $T_{2}$ and hence $T$ belongs to the image of $\varphi$.

Suppose that all horizontal strands on the top twist around the pole. Pick the strand that twists around the pole closest to the top and let $k$ be the endpoint on the top where it starts. Multiplying from the left by suitable $G_{i}$ as before, we can move the $k$ to 1 . Now the region between the strand from 1 on the top to the pole is a region of type $\mathrm{E}(6)$ and can be evacuated by Lemma 3.4. Now conjugate $T$ by $G_{0}$ to obtain that the horizontal strand from 1 on the top does not twist around the pole. If $n=2$, then $T$, having an even number of pole twists, must be $E_{2}$ and we are done. Suppose therefore, $n \geq 3$. If now all strands on the bottom twist around the pole, we multiply from the right by suitable $G_{i}$ to obtain a horizontal strand from the endpoint 1 at the bottom joint to the first pole twist from the bottom. After evacuation as before, in order not to relapse into the first horizontal strand at the top having a pole twist, we shift this strand away from 1, by premultiplying $T$ with $G_{2} G_{3}$ before conjugation by $G_{0}$. We now have horizontal lines on the top and bottom not going around the pole and we can apply the results of the previous paragraph.

Remarks 3.15. (i). The full algebra $\mathbf{K T}\left(\mathrm{D}_{n}\right)^{(2)}$ is generated by all $G_{i}, E_{i}$, for $i=1, \ldots n$, and the element $\rho$, consisting of one closed loop around the pole and vertical strands without crossings of which the one at the far left also has a pole twist. See Figure 3 ,

(ii). The algebra $\mathbf{K T}\left(\mathrm{D}_{n}\right)$ contains the algebra $\mathbf{K T}\left(\mathrm{A}_{n-1}\right)$ consisting of tangles which do not go around the pole. The elements $g_{i}, e_{i}$ of $\mathbf{B}\left(\mathrm{D}_{n}\right)$ for $i \geq 2$ satisfy the relations for $\mathbf{B}\left(\mathrm{A}_{n-1}\right)$ with the usual index increased by 1 . The proof of Theorem 3.14 also shows that the map $\varphi$ when restricted to $\mathbf{B}\left(\mathrm{A}_{n-1}\right)$ is a surjective homomorphism onto $\mathbf{K T}\left(\mathrm{A}_{n-1}\right)$. This was proved by similar means in 11. 
(iii). By Proposition 3.12 and Lemma 2.11 we can rewrite all closed tangles in $\mathbf{K T}\left(\mathrm{D}_{0}\right)^{(2)}$ to $\mathbb{Z}\left[\delta^{ \pm 1}\right]$-linear combinations of the identity, $\Xi^{+}$, and $\Theta$.

\section{The Brauer diagram algebra of type D}

In this section we introduce a variation of the original Brauer algebra using $(n, n)$ tangles of type D. It involves a variation of the $n$-connectors, known from the Brauer algebras in their classical sense, cf. [3, which we will recall first. The Brauer diagrams of type $\mathrm{D}_{n}$, to be introduced in Definition 4.4 encompass both the standard diagrammatic description of elements of the Weyl group $W\left(\mathrm{D}_{n}\right)$ and the diagrammatic elements of the (generalized) Temperley-Lieb algebra of type $\mathrm{D}_{n}$ introduced by Green in $[9$. Our goals are to show that the algebra defined on linear combinations of Brauer diagrams of type $\mathrm{D}_{n}$ is isomorphic to the Brauer algebra of type $\mathrm{D}_{n}$ as defined in 4 and to utilize this result to prove Theorem 1.1. For most of this final section, we use tangles and related diagrams of type $\mathrm{D}_{n}$, but at the end we discuss an alternative approach with the larger class of $(n, n)$ tangles of type $\mathrm{D}^{(2)}$.

Definition 4.1. An $n$-connector is a pairing on $2 n$ points into $n$ disjoint pairs.

These were used by Brauer in [3] to define an algebra over $\mathbb{Z}\left[\delta^{ \pm 1}\right]$, here called the Brauer diagram algebra. We take a basis of these $n$-connectors. An $n$-connector can be pictured by a diagram. The $2 n$ points are divided into two sets $\{1,2, \ldots, n\}$ and $\{\overline{1}, \overline{2}, \ldots, \bar{n}\}$ of points in the plane with each set on a horizontal line and point $i \in\{1, \ldots, n\}$ above $\bar{i}$. The points are connected by $n$ strands in the plane as determined by the pairing.

The product $a_{1} a_{2}$, for two $n$-connectors $a_{1}$ and $a_{2}$, is defined by stacking their diagrams with $a_{1}$ on top of $a_{2}$. Now identify node $\bar{i}$ of the bottom of $a_{1}$ with node $i$ of the top set of $a_{2}$, thus connecting the strands of $a_{1}$ and $a_{2}$. We find a new pairing between the nodes of the top of $a_{1}$ and the bottom of $a_{2}$. Besides a pairing, this can result in closed loops not connected to the top or bottom. When a composition gives $r$ closed loops, the new $n$-connector has coefficient $\delta^{r}$. So $a_{1} a_{2}=\delta^{r} a$ with $a$ the resulting $n$-connector. This multiplication is associative. In fact, these diagrams can be considered to be $(n, n)$-tangles in which the differences between over and under crossings are completely ignored. The multiplication is the same as the multiplication of the tangle after identification of over and under crossings in the resulting tangle. The classical Brauer diagram algebra, $\operatorname{BrD}\left(\mathrm{A}_{n-1}\right)$, over $\mathbb{Z}\left[\delta^{ \pm 1}\right]$ is the free $\mathbb{Z}\left[\delta^{ \pm 1}\right]$-module on the set of $n$-connectors with the described multiplication. We will now begin with the analogue for type D.

Definition 4.2. A decorated n-connector is an $n$-connector in which an even number of pairs is labelled 1. All other pairs are labelled 0. A pair labelled 1 will be called decorated. Denote $T_{n}$ the set of all decorated $n$-connectors. Denote $T_{n}^{0}$ the subset of $T_{n}$ of decorated $n$-connectors with no decorations and denote $T_{n}^{=}$the subset of $T_{n}$ of decorated $n$-connectors with at least one horizontal pairing.

We shall shortly (Definition 4.4) describe an algebra, $\operatorname{BrD}\left(\mathrm{D}_{n}\right)$, using certain decorated $n$-connectors together with some new parameters; we call it the Brauer diagram algebra of type $\mathrm{D}_{n}$. This algebra will turn out to be the image under the map $\psi$ assigning Brauer diagrams to tangles of type $\mathrm{D}_{n}$ by identifying over and under crossings, cf. Proposition 4.10, The definition will involve some collections 


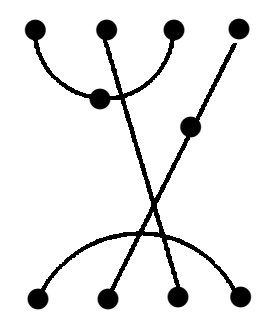

FiguRE 14. A decorated 4-connector with 2 decorated strands

of decorated $n$-connectors. For these purposes we need the sets $T_{n}^{0}$ and $T_{n}^{=}$defined above. Recall that $n ! !=(2 n-1)(2 n-3) \cdots 3 \cdot 1$, the product of the first $n$ odd integers.

Lemma 4.3. The sizes of the sets just defined are as follows. $\left|T_{n}\right|=2^{n-1} n !$ !, $\left|T_{n}^{0}\right|=n$ !!, and $\left|T_{n}^{=}\right|=2^{n-1}(n ! !-n !)$. Moreover, $\left|T_{n}^{=} \cap T_{n}^{0}\right|=n ! !-n !$.

Proof. The $2 n$ points can be paired in $n$ !! ways, so $\left|T_{n}^{0}\right|=n$ !!. Each strand can randomly be labelled 0 or 1 , except for the last one which is obliged to make the total number of decorations even. Hence, $\left|T_{n}\right|=2^{n-1} n !$ !.

To obtain a decorated $n$-connector without horizontal strands the $2 n$ points can be paired in $n$ ! ways. So there are $n ! !-n$ ! pairings which have at least one horizontal pair. These are all decorated $n$-connectors with at least one horizontal strand but without decorations, so $\left|T_{n}^{=} \cap T_{n}^{0}\right|=n ! !-n$ !. Again, these pairings can be decorated in $2^{n-1}$ ways, so $\left|T_{n}^{=}\right|=2^{n-1}(n ! !-n !)$.

For Brauer diagrams of type D, the role of the group $\left\langle\delta^{ \pm 1}\right\rangle$ for Brauer diagrams of type A will to some extent be taken over by the commutative monoid with presentation

$$
\Lambda=\left\langle\delta^{ \pm 1}, \xi, \theta \mid \xi^{2}=\delta^{2}, \xi \theta=\delta \theta, \theta^{2}=\delta^{2} \theta\right\rangle .
$$

In particular, we allow for decorated $n$-connectors to be multiplied with coefficients (central elements) from $\Lambda$. Notice $\Lambda=\left\langle\delta^{ \pm 1}\right\rangle\{1, \xi, \theta\}$.

Definition 4.4. A Brauer diagram of type $\mathrm{D}_{n}$ is the scalar multiple of a decorated $n$-connector with an element of $\Lambda$ belonging to $\left\langle\delta^{ \pm 1}\right\rangle\left(T_{n} \cup \xi T_{n}^{=} \cup \theta\left(T_{n}^{0} \cap T_{n}^{=}\right)\right)$. The Brauer diagram algebra of type $\mathrm{D}_{n}$, notation $\operatorname{BrD}\left(\mathrm{D}_{n}\right)$, is the $\mathbb{Z}\left[\delta^{ \pm 1}\right]$-linear span of all Brauer diagrams of type $\mathrm{D}_{n}$ with multiplication defined by $\mathbb{Z}\left[\delta^{ \pm 1}\right]$-bilinear extension of the multiplication of two Brauer diagrams $\lambda_{1} f_{1}$ and $\lambda_{2} f_{2}$, with $\lambda_{1}$, $\lambda_{2} \in\{1, \xi, \theta\}$ and $f_{1}, f_{2}$ decorated $n$-connectors, determined by the following five steps. Here, the product $\lambda_{1} f_{1} \lambda_{2} f_{2}$ is of the form $\lambda f$ where $f$ is a decorated $n$ connector and $\lambda \in \Lambda$.

(i) As before, draw the diagrams $f_{1}$ and $f_{2}$, stack them, and identify point $\bar{i}$ at the bottom of $f_{1}$ with $i$ at the top of $f_{2}$. The points at the top of $f_{1}$ will be identified with those for $f$ and similarly for the points at the bottom of $f_{2}$.

(ii) Determine the pairing of $f$ as before: for a point at the top of $f_{1}$ or the bottom of $f_{2}$, follow the strand until it ends in a point at the top of $f_{1}$ or the bottom of $f_{2}$. This results in a new pair for $f$.

(iii) Set $\lambda=\lambda_{1} \lambda_{2}$. For each straightening step in a concatenation of pairs as carried out in the previous step, check if the pattern shrunk to a straight horizontal line segment occurs as the left hand side of an equality in Figure 15. If so, 


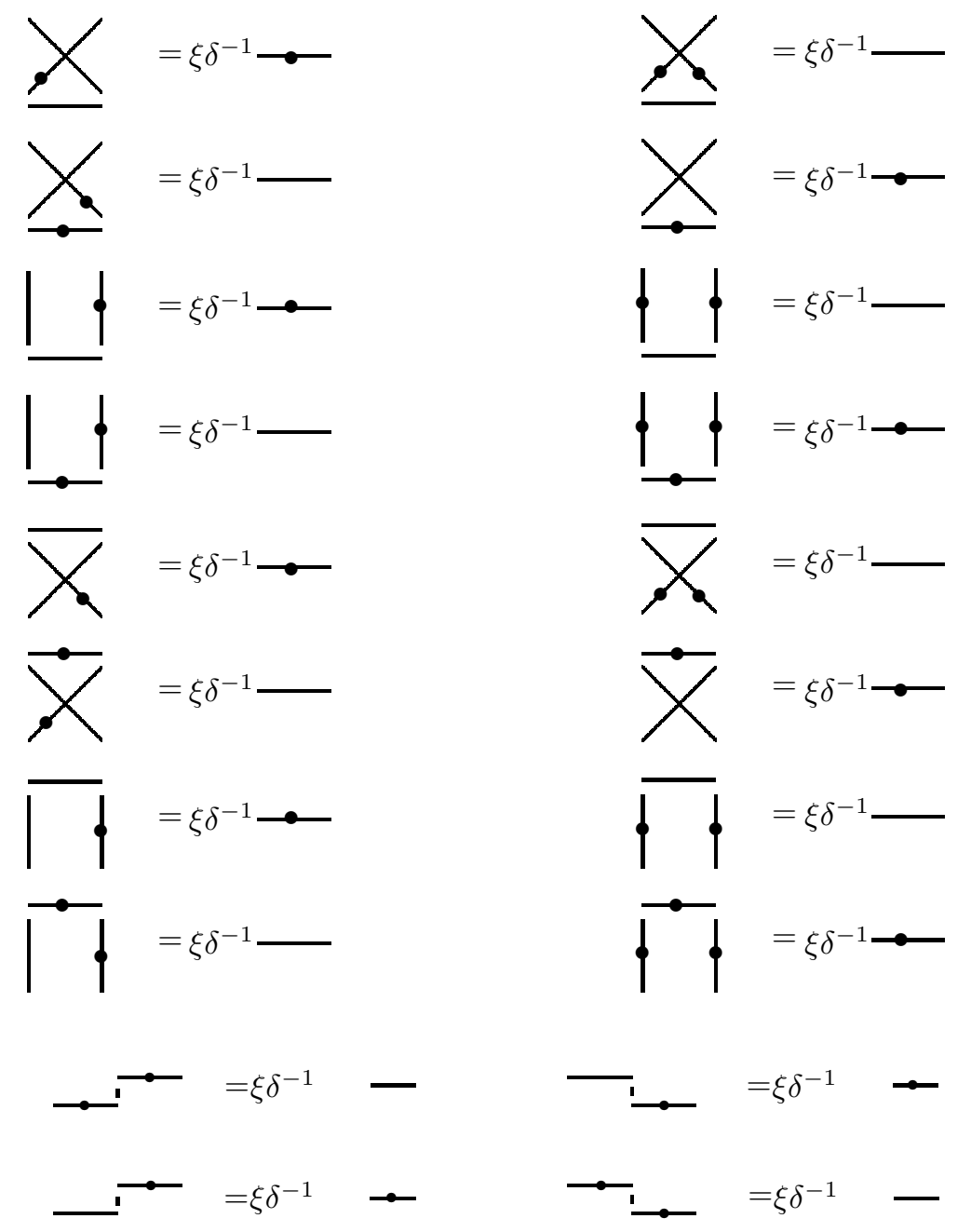

Figure 15. Rules for the composition of two vertical pairs and a horizontal pair in a product of two Brauer diagrams

multiply $\lambda$ by $\xi \delta^{-1}$; otherwise, $\lambda$ is not changed. (Compare with the left hand picture of Figure 16, this pattern as well as each triple of straight line segments forming a shape appearing in Figure 15 but whose decoration pattern does not appear in Figure 15, does not change $\lambda$.)

(iv) At this stage, only closed loops remain. Closed loops come from strands which have no endpoints in $f$. First simplify loops by removing crossings as in (iii), i.e. by use of Figure 15 (again, the configurations not appearing in the figure do not give $\left.\xi \delta^{-1}\right)$ and shrink them using the rules on the two bottom lines of Figure 15 (at this stage, factors $\xi \delta^{-1}$ may emerge). Next replace each closed loop without decoration by $\delta$ (that is, remove the loop and multiply $\lambda$ by $\delta$ ) and each pair of disjoint closed decorated loops by $\theta$. As the number of decorated pairs is even, what might remain is a simple decorated loop in the 
presence of a decorated pair; if so, undecorate the pair (i.e., give it label 0), remove the decorated loop and multiply $\lambda$ by $\theta \delta^{-1}$. (Compare with the right hand picture of Figure 16.)

$$
\oint=|\bigcirc|=\theta \delta^{-1}
$$

FiguRE 16. Two rules for decorated $n$-connectors

(v) If $\theta$ is a factor of $\lambda$, remove all decorations from $f$.

Thus, $\mathbf{B r D}\left(\mathrm{D}_{n}\right)$ is a free $\mathbb{Z}\left[\delta^{ \pm 1}\right]$-module. However, there is some indeterminacy in the description of the multiplication due to the order in which patterns are rewritten in (iii) and (iv). For instance in (iii), instead of applying the fifth rule from the top of the right column of Figure 15 to the top three strands of the right hand picture for $\xi$ in Figure 17, we could have applied the first rule of the same column of Figure 15 to the bottom three strands.

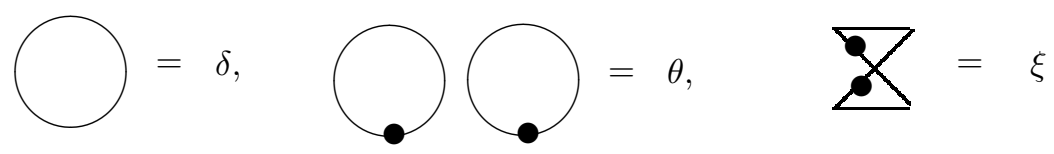

FigurE 17. Three closed loops occurring in Brauer diagrams

The proposition below shows that the multiplication on $\operatorname{BrD}\left(\mathrm{D}_{n}\right)$ is well defined by means of a $\mathbb{Z}\left[\delta^{ \pm 1}\right]$-linear map $\nu: \operatorname{Br}\left(\mathrm{D}_{n}\right) \rightarrow \operatorname{BrD}\left(\mathrm{D}_{n}\right)$ which we introduce first. Recall from [4] the set $\mathcal{A}_{0}$ of highest elements of $W$-orbits in $\mathcal{A}$, the set of all admissible sets of mutually orthogonal positive roots. We will use the action of $\operatorname{Br}\left(\mathrm{D}_{n}\right)$ on $\mathcal{A}$, denoted $\sigma$ in [4, Theorem 3.6(i)]. For $X \in \mathcal{A}_{0}$, we found a set $C_{W X}$ of nodes of $\mathrm{D}_{n}$ whose corresponding simple roots are orthogonal to $X$. We also defined $D_{Y}$ for $Y \in \mathcal{A}$ as a set of minimal length coset representatives of $N_{W}(Y)$ in $W$. By $\Phi$ we denote the root system of type $\mathrm{D}_{n}$. Its positive roots will be taken to be the vectors $\varepsilon_{j} \pm \varepsilon_{i}$ with $j>i$ in $\mathbb{R}^{n}$ with orthonormal basis $\varepsilon_{1}, \ldots, \varepsilon_{n}$. The simple roots are $\alpha_{1}=\varepsilon_{2}+\varepsilon_{1}$ and $\alpha_{i}=\varepsilon_{i}-\varepsilon_{i-1}$ for $i=2, \ldots, n$. If $\alpha$ is a root of $\Phi$, then $\alpha^{*}$ denotes its orthogonal mate, that is, the unique other positive root orthogonal to all roots orthogonal to $\alpha$, see [6, Definition 3.1]. If $\alpha=\varepsilon_{j}-\varepsilon_{i}$, then $\alpha^{*}=\varepsilon_{j}+\varepsilon_{i}$ and $\left(\alpha^{*}\right)^{*}=\alpha$. We write $r_{\alpha}^{*}$ for $r_{\alpha^{*}}$ and $r_{n}^{*}$ for the reflection with root $\alpha_{n}^{*}$ and, similarly, $e_{\alpha}^{*}$ for $e_{\alpha^{*}}$ and $e_{i}^{*}$ for $e_{\alpha_{i}}^{*}$. We will also use the natural permutation action of $W$ on $\{ \pm 1, \ldots, \pm n\}$, which maps $r_{\varepsilon_{j}-\varepsilon_{i}}$ to $(i, j)(-i,-j)$ and $r_{\varepsilon_{j}+\varepsilon_{i}}$ to $(i,-j)(-i, j)$.

Definition 4.5. By [4, Corollary 5.5], $\operatorname{Br}\left(\mathrm{D}_{n}\right)$ has a basis over $\mathbb{Z}\left[\delta^{ \pm 1}\right]$ consisting of the elements $u e_{X} z v$ with $X \in \mathcal{A}_{0}, u, v^{-1} \in D_{X}$, and $z \in W\left(C_{W X}\right)$. By 4, Lemma 1.2], either there is a root $\beta \in \Phi^{+}$such that both $\beta$ and $\beta^{*}$ belong to $X$, or $X$ is in the same $W$-orbit as $Y(t)$, for some $t \in\{1, \ldots,\lfloor n / 2\rfloor\}$, or $Y^{\prime}(n / 2)$ (in which case $n$ is even), where $Y(t)=\left\{\alpha_{n}, \alpha_{n-2}, \ldots, \alpha_{n-2 t+2}\right\}$ and $Y^{\prime}(n / 2)=$ $\left\{\alpha_{n}, \alpha_{n-2}, \ldots, \alpha_{4}, \alpha_{1}\right\}$, cf. [6. Section 3]. It will be more convenient for us to write 
the basis elements of the latter kind with $Y(t)$, respectively $Y^{\prime}(n / 2)$, instead of the highest element $X$. Observe that for this purpose $W\left(C_{W X}\right)$ needs to be replaced by $\left\langle r_{n}^{*}\right\rangle \times\left\langle r_{1}, \ldots, r_{n-2 t}\right\rangle$.

The Brauer diagram corresponding to $a=u e_{X} z v$ with $X=Y(t), Y^{\prime}(t)$, or $Y(t) \cup$ $Y(t)^{*}, u, v^{-1} \in D_{X}$, and $z=\left(r_{n}^{*}\right)^{k} z_{0}$ for some $k \in\{0,1\}$ and $z_{0} \in\left\langle r_{1}, \ldots, r_{n-2 t}\right\rangle$, is $\nu(a)=\lambda f$ where $\lambda \in \Lambda$ and the decorated $n$-connector $f$ are as follows. We first describe the horizontal strands of $f$. If $\varepsilon_{j}-\varepsilon_{i} \in u X=a \emptyset$, then $f$ has an undecorated horizontal strand pairing $i$ and $j$ at the top. Similarly, if $\varepsilon_{j}-\varepsilon_{i} \in v^{-1} X=a^{\mathrm{op}} \emptyset$ (where $a^{\text {op }}$ is obtained from $a$ by reversing an expression for $a$, see [4, Remark 5.7]) then $f$ has an undecorated horizontal strand pairing $\bar{i}$ and $\bar{j}$ at the bottom. Dually, if $\varepsilon_{j}+\varepsilon_{i} \in u X$ and $\varepsilon_{j}-\varepsilon_{i} \notin u X$, then $f$ has a decorated horizontal strand pairing $i$ and $j$ at the top, and if $\varepsilon_{j}+\varepsilon_{i} \in v^{-1} X$ but $\varepsilon_{j}-\varepsilon_{i} \notin v^{-1} X$, then $f$ has a decorated horizontal strand pairing $\bar{i}$ and $\bar{j}$ at the bottom.

Suppose $\beta^{*} \in X$ for some $\beta \in X$. Then, as $X$ is admissible, $\beta^{*} \in X$ for all $\beta \in X$. Put $\lambda=\theta \delta^{|X| / 2-2}$. (This is equal to $\left(\theta \delta^{-1}\right)^{|X| / 2}$.) As for the vertical strands of $f$, we connect $\bar{j}$ at the bottom with $j \notin v^{-1} X$ to $i$ at the top with an undecorated strand whenever $i= \pm u z v(j)$. This finishes the description of $\nu(a)$ in case $\beta, \beta^{*} \in X$ for some $\beta$.

Suppose now that $X=Y(t)$ for some $t \in\{1, \ldots,\lfloor n / 2\rfloor\}$. The treatment of $Y^{\prime}(n / 2)$ is the same as the treatment of $Y(n / 2)$ with the nodes 1 and 2 of $\mathrm{D}_{n}$ interchanged. Therefore, we do not discuss it further. Now $z=\left(r_{n}^{*}\right)^{k} z_{0}$ for some $k \in\{0,1\}$ and $z_{0} \in\left\langle r_{1}, \ldots, r_{n-2 t}\right\rangle$. Put $\lambda=\left(\xi \delta^{-1}\right)^{k}$. Finally, for the vertical strands of $f$, connect $\bar{j}$ at the bottom to $i$ at the top with an undecorated strand whenever $i=u z v(j)$ and with a decorated strand if $i=-u z v(j)$. This completes the definition of $\lambda$ and $f$ and hence of the Brauer diagram $\nu(a)$.

Observe that $\nu(a)$ is well defined. For instance, if $u$ and $u^{\prime}$ are elements of $W$ of minimal length with $u X=u^{\prime} X$, then $u z v$ and $u^{\prime} z v$ have the same action on the roots orthogonal to $v^{-1} X$, and similarly for $v$ and $v^{\prime}$ of minimal length with $v^{-1} X=v^{-1} X$, see [4, Lemma 4.8(i)]. Therefore $u z v(j)$ for $j$ not occurring as an index in $v^{-1} X$, does not depend on the choice of $D_{X}$.

So far we have defined $\nu(a)$ for $a$ belonging to a basis of $\operatorname{Br}\left(\mathrm{D}_{n}\right)$. By 4 , Theorem 1.1], the latter is a free $Z\left[\delta^{ \pm 1}\right]$-module, so $\nu$ can be extended by $Z\left[\delta^{ \pm 1}\right]$-linearity to a map $\operatorname{Br}\left(\mathrm{D}_{n}\right) \rightarrow \operatorname{BrD}\left(\mathrm{D}_{n}\right)$, which will also be denoted $\nu$.

Remarks 4.6. (i). For example, the Brauer diagrams $\nu\left(e_{i}\right)$ and $\nu\left(r_{i}\right)$ are as for $E_{i}$ and $G_{i}$ in Figures 10 and 11 after the twists around the pole have been replaced by decorations. Moreover, the version of $E_{i}$ with $i \geq 2$ in which both strands are decorated is equal to $\nu\left(e_{i}^{*}\right)$.

(ii). The Weyl group $W$ of type $\mathrm{D}_{n}$ can be diagrammatically described as follows by decorated diagrams having only vertical pairs: for $\beta=\varepsilon_{j} \pm \varepsilon_{i}$, the reflection $r_{\beta}$ is depicted by the diagram all of whose pairs are straight downwards and without decorations, except two vertical pairs connecting $i$ and $j$ at the top with $\bar{j}$ and $\bar{i}$ at the bottom, respectively, and both decorated if $\beta=\varepsilon_{j}+\varepsilon_{i}$, and neither decorated if $\beta=\varepsilon_{j}-\varepsilon_{i}$. Also, it is readily checked that the usual multiplication rules, with the one of the left picture in Figure 16 for the reduction of decorations if there are more than one per strand, suffice for the description of $W$ in terms of diagrams. This describes the restriction of $\nu$ to $W$.

(iii). Restriction of $\nu$ to $\operatorname{Br}\left(\mathrm{A}_{n-1}\right)$, where $\mathrm{A}_{n-1}$ stands for the set of nodes $\{2, \ldots, n\}$ of $\mathrm{D}_{n}$, gives an isomorphism between the Brauer algebra, $\operatorname{Br}\left(\mathrm{A}_{n-1}\right)$ 
and the Brauer diagram algebra $\operatorname{BrD}\left(\mathrm{A}_{n-1}\right)$, the $\mathbb{Z}\left[\delta^{ \pm 1}\right]$-linear span of all undecorated $n$-connectors (without scalars $\xi$ and $\theta$ ), see Figure 10, This fact has been known since the time of [13.

(iv). The equality involving the rightmost closed loop of Figure 17 is a consequence of the fifth rule from the top in the right column of Figure 15 (applied to the top three strands with a decoration on each of the two diagonal strands). For, after removing the crossing by that rule, a closed undecorated loop remains, so the whole loop can be replaced by $\left(\xi \delta^{-1}\right) \delta$, which is equal to $\xi$. Similarly the top rule on the right can be used.

(v). The diagrams given in the two last rows of Figure 15, where the common endpoint of the two horizontal strands is in the middle, suffice for describing all computations involving multiple horizontal strands. An example is given in Figure 18 .

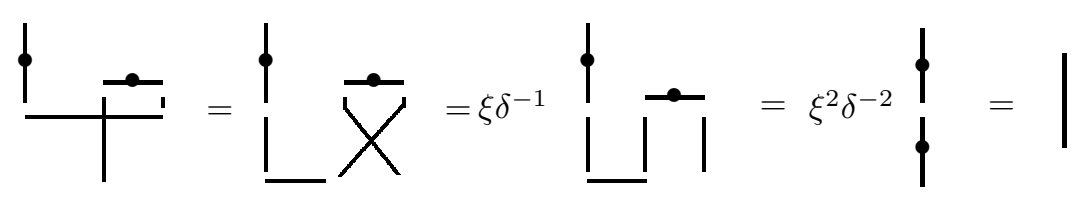

FiguRE 18. Multiplication involving horizontal pairs

It is straightforward to check that when two horizontal strands have their common endpoint on the left, the scalar $\xi \delta^{-1}$ only comes in when precisely one of the two strands has a decoration. When two horizontal strands have their common node on the right, the scalar $\xi$ nevers appears, as in Figure 18 .

(vi) The map $\nu$ satisfies the following identities for positive roots $\alpha$, respectively $\alpha$ and $\beta$, with $|(\alpha, \beta)|=1$.

$$
\nu\left(r_{\alpha}^{*} e_{\alpha}\right)=\xi \delta^{-1} \nu\left(e_{\alpha}\right), \quad \nu\left(r_{\alpha} r_{\alpha}^{*} e_{\beta}\right)=\xi \delta^{-1} \nu\left(e_{\beta}^{*} e_{\alpha} e_{\beta}\right) .
$$

Indeed, these equations hold for $\alpha=\alpha_{2}$, respectively $\alpha=\alpha_{2}$ and $\beta=\alpha_{3}$, and follow from these case by conjugation with a suitable Weyl group element (observe that the $\xi$ factors of $\nu\left(r_{i} e_{\alpha}\right)$ and $\nu\left(e_{\alpha} r_{i}\right)$ agree for every node $i$, so for each $w \in W$ with $w \alpha_{1}=\alpha$, we have $\left.\nu\left(e_{\alpha}\right)=\nu(w) \nu\left(e_{1}\right) \nu\left(w^{-1}\right)\right)$.

Lemma 4.7. For $i, j, k \in\{1, \ldots, n\}$, denote by $f_{i j}^{k}$ the decorated $n$-connector all of whose pairs are of the form $\{x, \bar{x}\}$ except for $x \in\{i, j\}$, where the pairs are $\{i, j\}$ and $\{\bar{i}, \bar{j}\}$, and of which only the top horizontal pair $\{i, j\}$ and the vertical pair $\{k, \bar{k}\}$ are labeled 1 . Similarly, let $g_{i j}^{k}$ be as $f_{i j}^{k}$ but with the bottom horizontal pair $\{\bar{i}, \bar{j}\}$ labeled 1 instead of $\{i, j\}$. Then, for $i<j$ and $k \notin\{i, j\}$,

$$
\nu\left(r_{\alpha} r_{\alpha}^{*} e_{\beta}\right)= \begin{cases}f_{i j}^{k} & \text { if } \alpha=\varepsilon_{k}-\varepsilon_{i} \text { and } \beta=\varepsilon_{j}-\varepsilon_{i} \\ \xi \delta^{-1} f_{i j}^{k} & \text { if } \alpha=\varepsilon_{k}-\varepsilon_{j} \text { and } \beta=\varepsilon_{j}-\varepsilon_{i} \\ g_{i j}^{k} & \text { if } \alpha=\varepsilon_{k}-\varepsilon_{i} \text { and } \beta=\varepsilon_{j}+\varepsilon_{i} \\ \xi \delta^{-1} g_{i j}^{k} & \text { if } \alpha=\varepsilon_{k}-\varepsilon_{j} \text { and } \beta=\varepsilon_{j}+\varepsilon_{i}\end{cases}
$$

Proof. For $i=1, j=2, k=3$, the formulas follow from straightforward computations. For instance, for the second line, with $\alpha=\varepsilon_{3}-\varepsilon_{1}, \beta=\varepsilon_{3}-\varepsilon_{2}$, we find $r_{\alpha} r_{\alpha}^{*} e_{\beta}=\left(r_{3} r_{2} r_{3}\right)\left(r_{3} r_{1} r_{3}\right) e_{3}=r_{3} r_{2} r_{1} e_{3}=r_{2} r_{1} e_{3} r_{3}^{*}$ and so $\nu\left(r_{\alpha} r_{\alpha}^{*} e_{\beta}\right)=$ $\xi \delta^{-1} \nu\left(r_{2} r_{1} e_{3}\right)=\xi \delta^{-1} f_{23}^{1}$ by the definition of $\nu$. 
For general $i<j$, the proof can be finished by induction on the sum of the distances between $i, j$, and $k$.

Proposition 4.8. The $\mathbb{Z}\left[\delta^{ \pm 1}\right]$-algebra $\operatorname{BrD}\left(\mathrm{D}_{n}\right)$ is well defined and the linear map $\nu: \operatorname{Br}\left(\mathrm{D}_{n}\right) \rightarrow \operatorname{BrD}\left(\mathrm{D}_{n}\right)$ is an isomorphism.

Proof. By reversing the above definition of $\nu(a)$, we find that each Brauer diagram is of the form $\delta^{k} \nu(a)$ for some $k \in \mathbb{Z}$ and some monomial $a$ in $\operatorname{Br}\left(\mathrm{D}_{n}\right)$, so $\nu$ is surjective. As a $\mathbb{Z}\left[\delta^{ \pm 1}\right]$-module, the Brauer diagram algebra $\mathbf{B r D}\left(\mathrm{D}_{n}\right)$ is free with basis $T_{n} \cup \xi T_{n}^{=} \cup \theta\left(T_{n}^{0} \cap T_{n}^{=}\right)$. Its dimension is $\left|T_{n}\right|+\left|T_{n}^{=}\right|+\left|T_{n}^{0} \cap T_{n}^{=}\right|$. By Lemma4.3 this number equals $d(n)$, the dimension of the free $\mathbb{Z}\left[\delta^{ \pm 1}\right]$-algebra $\operatorname{Br}\left(\mathrm{D}_{n}\right)$, cf. [4, Theorem 1.1], and so $\nu$ is bijective.

There is a unique multiplication on $\operatorname{BrD}\left(\mathrm{D}_{n}\right)$ such that $\nu$ is an isomorphism of $\mathbb{Z}\left[\delta^{ \pm 1}\right]$-algebras. We claim that this multiplication satisfies the rules of Definition 4.4. For Steps (i) and (ii), as well as the $\delta$ part of (iv), the rules coincide with the laws known for $\mathrm{A}_{n-1}$ and settle the multiplication up to decorations and the coefficient in $\Lambda$. To substantiate the claim for these two steps, consider the $\mathbb{Z}\left[\delta^{ \pm 1}\right]$ algebra homomorphism $\pi$ on $\mathbf{B r}\left(\mathrm{D}_{n}\right)$ determined by $\pi\left(r_{1}\right)=r_{2}, \pi\left(e_{1}\right)=e_{2}$ and fixing the other generators. The homomorphism $\pi$ projects $\operatorname{BrD}\left(\mathrm{D}_{n}\right)$ onto its subalgebra generated by $r_{i}$ and $e_{i}$ for $i>1$. By inspection of the defining relations, it is clear that this subalgebra is a homomorphic image of $\mathbf{B r}\left(\mathrm{A}_{n-1}\right)$ (with a shift of 1 in the indices of the usual generators). The decorated $n$-connectors that are $\nu$-images of monomials from this subalgebra are precisely those belonging to the usual Brauer diagram algebra of undecorated $n$-connectors (appearing here as the $n$-connectors all of whose labels are 0 , cf. Remark 4.6 (iii)). Therefore, the restriction of $\nu$ to the image of $\pi$ is surjective onto the Brauer diagram algebra $\operatorname{BrD}\left(\mathrm{A}_{n-1}\right)$, and so the image of $\pi$ is a subalgebra of $\operatorname{Br}\left(\mathrm{D}_{n}\right)$ isomorphic to $\operatorname{Br}\left(\mathrm{A}_{n-1}\right)$. In terms of Brauer diagrams, $\nu \circ \pi \circ \nu^{-1}$ sends a Brauer diagram $\lambda f$, where $\lambda \in \Lambda$ and $f$ is a decorated $n$-connector, to $\pi(\lambda) \pi(f)$, where $\pi(f)$ is obtained from $f$ by removing the decorations, and $\pi(\lambda)$ is determined by $\pi(\delta)=\pi(\xi)=\delta$ and $\pi(\theta)=\delta^{2}$. In this light, all that is needed to verify is that, for monomials $a, b \in \operatorname{BrM}\left(\mathrm{D}_{n}\right)$, the decoration of the decorated $n$-connector $f$ and the coefficient $\lambda$ of the result $\lambda f$ of the multiplication of $\nu(a)$ and $\nu(b)$ according to Definition 4.4 coincide with those of $\nu(a b)$.

The homomorphism $\pi$ also shows that the left rule of Figure 16 applies: a label 1 on a vertical strand from top node $h$ to bottom node $\bar{k}$ means that the central part (the element $u z v$ in the notation of Definition 4.5) of the corresponding monomial $u e_{X} z v$ maps $k$ to $-h$, and so two consecutive labels 1 on the same strand cancel. For Step (iii) of Definition 15, we need to verify the rules of Figure 15, The four rules on the two bottom lines are consequences of the others: taking the vertical pairs into account, we can view the left hand sides of each of these as a $U$-shaped triple of line segments followed or preceeded by a similar triple upside down whose neighboring vertical pair is identified with the neighboring vertical pair of the first triple. By the rule for the $U$-shaped triple, read backwards, and next for the second triple, read forward, the presence of a $\xi \delta^{-1}$ factor can be determined. See Figure 19 for an example.

If $a$ and $b$ are outside the ideal generated by the $e_{i}$, they belong to $W\left(\mathrm{D}_{n}\right)$ up to multiples from $\left\langle\delta^{ \pm 1}\right\rangle$; in this case $\nu(a)$ and $\nu(b)$ only have vertical pairs and the decoration rules of Definition 4.4 are easily seen to hold. Also, when no bottom ends of a crossing of two vertical pairs of $\nu(a)$ match the ends of a top horizontal 


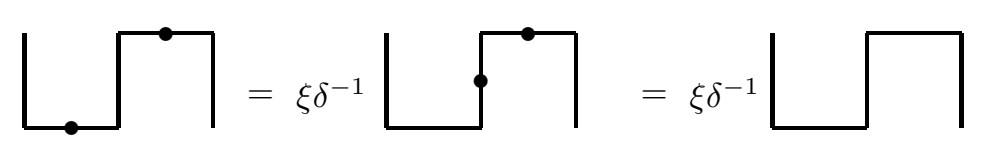

Figure 19. Second rule at the left from below of Figure 15 obtained as a consequence of two earlier rules

pair of $\nu(b)$, no changes in the coefficient $\lambda$ occurs. We will use these observations to reduce the configurations to be considered.

Both the rules of the figure and the relations defining $\operatorname{Br}\left(\mathrm{D}_{n}\right)$ are closed under opposition, i.e., reflecting the picture in a horizontal mirror and reversing an expression for a word in the generators of $\operatorname{Br}\left(\mathrm{D}_{n}\right)$. Therefore, it suffices to consider the rules of Figure 15] in which the horizontal strands belong to $\nu(b)$ and the two vertical strands belong to $\nu(a)$.

We now show that the rules whose vertical pairs (belonging to $\nu(a)$ ) cross, follow from those without crossings. The latter will be called $U$ rules, a name reminding us of their shapes. Take any left hand side of an equality with the horizontal pair in $\nu(b)$ and crossing vertical pairs in $\nu(a)$, insert straight line segments above the top corners and move the decoration, if present, on the horizontal pair towards the vertical pair ending at the left (this is a $U$ rule); next move all decorations up towards the top vertical line segments by the rules for $W\left(\mathrm{D}_{n}\right)$. At the bottom, we have a triple of undecorated pairs; now the rules for undecorated pairs are those for the Brauer diagram algebra of type $\mathrm{A}_{n-1}$, and allow us to replace the triple of line segments by a single horizontal pair, and we can finish by the applying $U$ rules. See Figure 20 for an example.

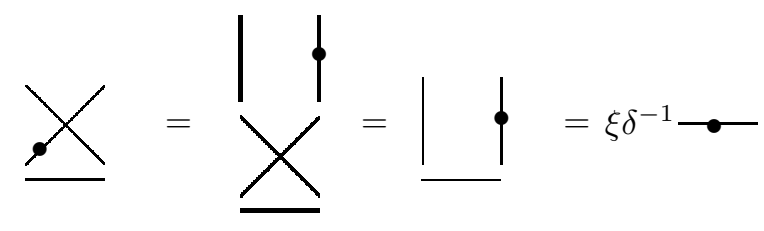

Figure 20. Derivation of a multiplication rule with a crossing

Next we verify the third and fourth rule from the top of Figure15. Suppose that the left hand side of a $U$ rule in one of these two rows has left bottom corner at node $\bar{i}$ and right bottom corner at node $\bar{j}$, so that $i<j$. Then, by pre-multiplications with suitable reflections $r_{\gamma}$ for $\gamma=\varepsilon_{k}-\varepsilon_{i}$ and $\gamma=\varepsilon_{j}-\varepsilon_{k}$, the vertical pairs of the triple above $\bar{i}$ and $\bar{j}$ can be made straight, that is, equal to $\{i, \bar{i}\}$ and $\{j, \bar{j}\}$, respectively, without changing the $\xi$ factors. Set $\beta=\varepsilon_{j}-\varepsilon_{i}$ or $\beta=\varepsilon_{j}+\varepsilon_{i}$ according to whether the horizontal pair in the figure has label 0 or 1 .

First consider the left hand side of the equation in rows 3 and 4 in the right column of Figure 15. Here $a=a^{\prime} r_{\beta} r_{\beta}^{*}$ and $b=e_{\beta} b^{\prime}$ for certain monomials $a^{\prime}, b^{\prime}$ in $\operatorname{BrM}\left(\mathrm{D}_{n}\right)$ and $\beta=\varepsilon_{j} \pm \varepsilon_{i}$. Now $\nu(a b)=\nu\left(a^{\prime}\right) \nu\left(r_{\beta} r_{\beta}^{*} e_{\beta}\right) \nu\left(b^{\prime}\right)=\nu\left(a^{\prime}\right) \nu\left(r_{\beta}^{*} e_{\beta}\right) \nu\left(b^{\prime}\right)=$ $\xi \delta^{-1} \nu\left(a^{\prime}\right) \nu\left(e_{\beta}\right) \nu\left(b^{\prime}\right)=\xi \delta^{-1} \nu\left(a^{\prime}\right) \nu(b)$, which proves the rules on rows 3 and 4 in the right column.

To deal with the third and fourth rule from the top left of Figure 15, consider the left hand side of a $U$ rule with vertical pairs $\{i, \bar{i}\}$ and $\{j, \bar{j}\}$, where $i<j$ as 
before, and suppose that $\{j, \bar{j}\}$ is the only vertical pair of the triple with label 1 . As only one decoration in $\nu(a)$ is visible and there are an even number, there must be another decorated pair. Without loss of generality, the second decoration can be taken to be on a vertical pair with bottom end point, say $\bar{k}$ distinct from $\bar{i}$ and $\bar{j}$. For, if there would be a bottom horizontal pair with a bottom end node $\bar{k}$, the monomial $a$ could be rewritten so as to end with the monomial $a_{2}=r_{\gamma} r_{\gamma}^{*}$ with $\gamma= \pm\left(\varepsilon_{k}-\varepsilon_{j}\right)$, so that $\nu\left(a_{2}\right)$ is the identity except that the vertical pairs $\{j, \bar{j}\}$ and $\{k, \bar{k}\}$ are labeled 1 ; as the pair with label 1 of $\nu\left(a_{2}\right)$ ending in $\bar{k}$ does not change and neither does its label, the multiplication rule will be as required for $a$ once it holds for $a_{2}$ instead of $a$. Now let $\beta=\varepsilon_{j} \pm \varepsilon_{i}$ as before and set $\alpha=\varepsilon_{j}-\varepsilon_{k}$. There are monomials $a^{\prime}, b^{\prime} \in \operatorname{BrM}\left(\mathrm{D}_{n}\right)$ such that $a=a^{\prime} r_{\alpha} r_{\alpha}^{*} e_{\beta}$ and $b=e_{\beta} b^{\prime}$. By Lemma 4.7 $\nu(a b)=\nu\left(a^{\prime}\right) \nu\left(r_{\alpha}^{*} r_{\alpha} e_{\beta}\right) \nu\left(b^{\prime}\right)=\xi \delta^{-1} \nu\left(a^{\prime}\right) f_{i j}^{k} \nu\left(b^{\prime}\right)$ which establishes the remaining rules of Figure 15. Those $U$ rules which are not present (because they produce no factor $\xi \delta^{-1}$ ), can be treated in the same way. This ends the verification of Step (iii).

The parts of Step (iv) not involving $\theta$ are as before or familiar from the case $\mathrm{A}_{n-1}$. The simplest instance of the $\theta$ related rule occurs for $a=e_{1}$ and $b=e_{2}$, when $\theta \delta^{-1} \nu\left(e_{1}\right)=\nu\left(e_{1} e_{2}\right)$. By conjugation with Weyl group elements, it generalizes to $\theta \delta^{-1} \nu\left(e_{\beta}\right)=\nu\left(e_{\beta} e_{\beta}^{*}\right)$ for every positive root $\beta$, and, after multiplication with suitable reflections, this accounts for the last part of (iv).

As for Step (v), by definition of $\nu$, the $n$-connector of $\nu(a)$ has no decorations in the presence of a scalar factor $\theta$. The rule is in accordance with $\theta \nu\left(e_{\beta}^{*}\right)=\theta \nu\left(e_{\beta}\right)$.

We conclude that the rules used in Definition 4.4 are satisfied by the multiplication turning $\nu$ into an isomorphism. This establishes that the latter is well defined.

Remarks 4.9. (i). The inverse of $\nu$ can be described effectively. Given the Brauer diagram $\lambda f$ of type $\mathrm{D}_{n}$ with decorated $n$-connector $f$ and $\lambda \in\{1, \xi, \theta\}$, we describe how to find a monomial $a=u e_{X} z v \in \operatorname{Br}\left(\mathrm{D}_{n}\right)$ such that $\nu(a) \in\left\langle\delta^{ \pm 1}\right\rangle \lambda f$. As $\theta \nu\left(u e_{X} z v\right)=\delta^{2-|X|} \nu\left(u e_{X^{\prime}} z v\right)$ where $X^{\prime}=\left\{\beta, \beta^{*} \mid \beta \in X\right\}$ if $X$ has no mutually orthogonal mates, and as $\xi \nu\left(u e_{X} z v\right)=\delta \nu\left(u e_{X} r_{n}^{*} z v\right)$ whenever $\beta \in X$ and $X$ is equal to some $Y(t)$ or to $Y^{\prime}(n / 2)$, it suffices to consider the case where $\lambda=1$. Observe that $a \emptyset=u X$ and $a^{\mathrm{op} \emptyset}=v^{-1} X$ can be read off from the horizontal strands at the top and bottom of $f$, respectively. As $u, v^{-1} \in D_{X}$ and $D_{X}$ is fixed, this determines $u$ and $v$ uniquely. Finally, $z$ is determined by the vertical strands of $\nu\left(u^{-1}\right) f \nu\left(v^{-1}\right)$.

(ii). Two special cases of Brauer diagrams of type $\mathrm{D}_{n}$ are known. First those without horizontal pairs, see Remark 4.6(ii). Second, in 9] diagrams for the TemperleyLieb algebra of type $\mathrm{D}_{n}$ are considered. As discussed in [6, Remark 5.2], the Temperley-Lieb algebra is a subalgebra of $\operatorname{Br}\left(\mathrm{D}_{n}\right)$, and by the fact that $\nu$ is an isomorphism, also of $\operatorname{BrD}\left(\mathrm{D}_{n}\right)$. The diagrams appearing in [9] are precisely the Brauer diagrams without crossings (take into account that $\xi$ also contains a crossing and so does not appear), that is, these are compositions of the $\nu\left(e_{i}\right)$.

Recall the definition of $G_{i}$ and $E_{i}$ from Figures 10 and 11. Write $G_{i}^{-1}$ for the same tangle as $G_{i}$ but with the over crossing changed into an under crossing. There is a natural map assigning to a tangle in $\mathcal{U}_{n}$ that is a composition of the basic tangles $G_{i}, G_{i}^{-1}$, and $E_{i}$, a Brauer diagram of type $\mathrm{D}_{n}$. For such a $t \in \mathcal{U}_{n}$, let $\psi(t)$ be the Brauer diagram of type $\mathrm{D}_{n}$ obtained by identifying over and under crossings and by replacing twists around the pole of a strand by addition of $1 \bmod 2$ (that 
is, applying a change of decoration) to the strand. This means the element $\psi(t)$ is the product in $\operatorname{BrD}\left(\mathrm{D}_{n}\right)$ of the Brauer diagrams arising by substitution of $\nu\left(r_{i}\right)$ for each $G_{i}$ and for each $G_{i}^{-1}$ and of $\nu\left(e_{i}\right)$ for each $E_{i}$ occurring in an expression of $t$ as a composition of basic tangles. By construction, $\psi$ is a homomorphism of monoids.

The identification of over and under crossings means that the scalars $\Xi^{ \pm}$and $\Theta$ are replaced by the scalars $\xi$ and $\theta$, respectively. Under $\psi$, the left and right hand sides of Figure 13 become the rule at the right top of Figure 15 but for the isolated horizontal strand at the bottom.

Proposition 4.10. The map $\psi$ induces an $R$-equivariant homomorphism of rings $\mathbf{K T}\left(\mathrm{D}_{n}\right) \rightarrow \mathbf{B r D}\left(\mathrm{D}_{n}\right)$, also denoted by $\psi$. It satisfies $\psi \circ \varphi=\nu \circ \mu$.

Proof. Denote by $\mathcal{U}_{n}^{(0)}$ the set of $(n, n)$-tangles in $\mathcal{U}_{n}$ that are a composition of the basic tangles $G_{i}, G_{i}^{-1}$, and $E_{i}$. As $R /(\{l-1, m\}) R \cong \mathbb{Z}\left[\delta^{ \pm 1}\right]$, the map $\psi$ can be linearly extended to a map $R\left[\mathcal{U}_{n}^{(0)}\right] \rightarrow \operatorname{BrD}\left(\mathrm{D}_{n}\right)$ with $l-1$ and $m$ in its kernel.

We claim that $\psi$ factors through $\mathbf{K T}\left(\mathrm{D}_{n}\right)$ because the relations of Definition 2.6 hold for the tangles replaced by their images in $\operatorname{BrD}\left(\mathrm{D}_{n}\right)$. The double twist relation is equivalent with the rule in $\operatorname{BrD}\left(\mathrm{D}_{n}\right)$ that two decorations on one strand cancel each other. The Kauffman skein relation (i) holds as by $m=0$ the relation reduces to the equality of over and under crossings. The two partial diagrams satisfying the commuting relation (ii) are both mapped to the same partial diagram containing two decorated vertical strands. The self-intersection relations (iii) hold in $\operatorname{BrD}\left(\mathrm{D}_{n}\right)$ with $l=1$. The idempotent relation (iv) carries over to the same relation in $\operatorname{BrD}\left(\mathrm{D}_{n}\right)$. Both sides of the pole-related self-intersection relations (v) and (vi) are mapped to the same multiple of a decorated $n$-connector by a scalar $\xi \delta^{-1}$. (For (v), use the first and fifth rule in the left column of Figure 15, for (vi) use the second and the sixth from the same column.) The first closed pole loop relation (vii) is covered by rewriting the images under $\psi$ of both sides by use of $\theta$. Further details are left to the reader. It may be worthy of note that the self-intersection relations (iii) only demand that $l^{2}=1$ and that the choice $l=1$ corresponds to the complete removal of self-intersections as in Reidemeister I. Therefore, the defining relations for $\mathbf{K T}\left(\mathrm{D}_{n}\right)$ are in the kernel of $\psi$. As $\psi$ is a homomorphism of monoids, even the ideal of $\mathbb{Z}\left[\delta^{ \pm 1}\right]\left[\mathcal{U}_{n}^{(0)}\right]$ generated by the defining relations for $\mathbf{K T}\left(\mathrm{D}_{n}\right)$ is in the kernel of $\psi$, and so $\psi$ factors through $\mathbf{K T}\left(\mathrm{D}_{n}\right)$, as claimed.

In view of Theorem 3.14 $\mathbf{K T}\left(\mathrm{D}_{n}\right)$ is linearly spanned by monomials in $G_{i}$ and $E_{i}$ and so the map $\psi$ is defined on all of $\mathbf{K T}\left(\mathrm{D}_{n}\right)$. We conclude that $\psi$ is well defined as an $R$-equivariant ring homomorphism $\mathbf{K T}\left(\mathrm{D}_{n}\right) \rightarrow \mathbf{B r D}\left(\mathrm{D}_{n}\right)$.

Now $\psi\left(G_{i}\right)=\nu\left(r_{i}\right)$ and $\psi\left(E_{i}\right)=\nu\left(e_{i}\right)$ so $\psi \varphi\left(g_{i}\right)=\nu \mu\left(g_{i}\right)$ and $\psi \varphi\left(e_{i}\right)=\nu \mu\left(e_{i}\right)$ for $i=1, \ldots, n$. In particular, $\psi \circ \varphi$ and $\nu \circ \mu$ are two $R$-equivariant ring homomorphisms agreeing on the generators of $\mathbf{B}\left(\mathrm{D}_{n}\right)$, so they coincide. As $\nu$ and $\mu$ are surjective, so is $\psi \circ \varphi$. Consequently, $\psi$ is surjective.

This proves Theorem 1.1(i) and (iv). We are ready for the last main result, which settles Theorem 1.1(iii). Notice Theorem[1.1(ii) is immediate from Proposition 4.8.

Theorem 4.11. The map $\varphi: \mathbf{B}\left(\mathrm{D}_{n}\right) \rightarrow \mathbf{K T}\left(\mathrm{D}_{n}\right)$ is an isomorphism of R-algebras. Both algebras are free of dimension $d(n)$, the dimension of the Brauer algebra of type $\mathrm{D}_{n}$. The tensor products of these algebras with $\mathbb{Q}(l, \delta)$ over $R$ are semisimple. 
Proof. In view of surjectivity of $\psi$, the dimension of $\mathbf{K T}\left(\mathrm{D}_{n}\right)$ is at least $d(n)$. By an argument similar to the one for [6, Lemma 4.2] applied to $\nu^{-1} \circ \psi$, we see that $\mathbf{K T}\left(\mathrm{D}_{n}\right)$ is a free $R$-module. But $\varphi$ is surjective by Theorem [3.14, and $\mathbf{B}\left(\mathrm{D}_{n}\right)$ is free of dimension $d(n)$ as well, by [6, Theorem 1.1]. Therefore, $\varphi$ is an isomorphism. Also by [6. Theorem 1.1], $\mathbf{B}\left(\mathrm{D}_{n}\right)$, when tensored with $\mathbb{Q}(l, \delta)$, is semi-simple. This implies the semisimplicity statement.

Remarks 4.12. (i). Our work also proves the corresponding result for $\mathbf{B}\left(\mathrm{A}_{n-1}\right)$ known from [11: the BMW algebra $\mathbf{B}\left(\mathrm{A}_{n-1}\right)$ is isomorphic to the tangle algebra $\mathbf{K T}\left(\mathrm{A}_{n}\right)$ given via the map $\varphi$ of Proposition 4.10. The dimension is $n$ !!, the same as the dimension of the Brauer algebra of type $A_{n-1}$. The proof of these results obtainable from the arguments in the proof of Theorem4.11 differs from the original one and is far easier than the one for $\mathrm{D}_{n}$ as there is no $e_{i}^{*}$, no $\Xi^{+}$, and no $\Theta$.

(ii). In Definition 2.6 we encountered the $R$-algebra $\mathbf{K T}\left(\mathrm{D}_{n}\right)^{(2)}$ properly containing $\mathbf{K T}\left(\mathrm{D}_{n}\right)$. There are corresponding versions of the other algebras of Theorem 1.1 and the maps such that the following diagram commutes.

$$
\begin{array}{ccc}
\mathbf{B}\left(\mathrm{D}_{n}\right)^{(2)} & \stackrel{\mu^{(2)}}{\longrightarrow} & \mathbf{B r}\left(\mathrm{D}_{n}\right)^{(2)} \\
\varphi^{(2)} \downarrow & & \downarrow \nu^{(2)} \\
\mathbf{K T}\left(\mathrm{D}_{n}\right)^{(2)} & \stackrel{\psi^{(2)}}{\longrightarrow} & \mathbf{B r D}\left(\mathrm{D}_{n}\right)^{(2)}
\end{array}
$$

Here $\mathbf{B}\left(\mathrm{D}_{n}\right)^{(2)}$ denotes the algebra over $R\left[\Xi^{+}, \Theta\right]$, specified by the relations of Lemma 2.11, that is generated by $g_{i}$ and $e_{i}(i=1, \ldots, n)$ subject to the relations known for $\mathbf{B}\left(\mathrm{D}_{n}\right)$ from Definition 2.1 and

$$
\Xi^{+} e_{1}=\delta g_{2} e_{1}, \quad \quad \quad \Theta e_{1}=\delta e_{1} e_{2} .
$$

Similarly, $\operatorname{Br}\left(\mathrm{D}_{n}\right)^{(2)}$ denotes the algebra over $\mathbb{Z}[\Lambda]$ generated by $r_{i}$ and $e_{i}(i=$ $1, \ldots, n)$ subject to the relations known for $\mathbf{B r}\left(\mathrm{D}_{n}\right)$ in [4, Table 1] and

$$
\xi e_{1}=\delta r_{2} e_{1}, \quad \theta e_{1}=\delta e_{2}=\delta e_{1} e_{2} .
$$

The algebra $\operatorname{BrD}\left(\mathrm{D}_{n}\right)^{(2)}$ is linearly spanned over $\mathbb{Z}[\Lambda]$ by $T_{n}$. It is not free over this ring, but free with basis $T_{n} \cup \xi T_{n} \cup \theta T_{n}^{0}$ over $\mathbb{Z}\left[\delta^{ \pm 1}\right]$, and so its dimension is $\left(2^{n}+1\right) n$ !! if $n \geq 1$. For $n=0$, the algebra $\operatorname{BrD}\left(\mathrm{D}_{n}\right)^{(2)}$ coincides with $\mathbb{Z}[\Lambda]$ which is free of dimension 3 over $\mathbb{Z}\left[\delta^{ \pm 1}\right]$.

The following relations hold in $\operatorname{Br}\left(\mathrm{D}_{n}\right)$ for all $\alpha, \beta \in \Phi^{+}$.

$$
r_{\alpha^{*}} e_{\alpha} e_{\beta}=e_{\alpha} r_{\beta^{*}} e_{\beta}, \quad e_{\alpha^{*}} e_{\alpha} e_{\beta}=e_{\alpha} e_{\beta^{*}} e_{\beta},
$$

They can be used to show that $\mathbf{B r D}\left(\mathrm{D}_{n}\right)$ embeds in $\mathbf{B r D}\left(\mathrm{D}_{n}\right)^{(2)}$, and that $\mathbf{B}\left(\mathrm{D}_{n}\right)$ embeds in $\mathbf{B}\left(\mathrm{D}_{n}\right)^{(2)}$. All maps in the diagram are $R$-equivariant ring homomorphisms. The vertical maps $\varphi^{(2)}$ and $\nu^{(2)}$ are algebra isomorphisms again. The action $\sigma$ of $\operatorname{BrM}\left(\mathrm{D}_{n}\right)$ on $\mathcal{A}$, see [4, Theorem 3.6(i)] extends to an action of $\operatorname{BrM}\left(\mathrm{D}_{n}\right)^{(2)}$ on $\mathcal{A}$ with

$$
\xi X=X, \quad \theta X=X \cup\left\{\beta^{*} \mid \beta \in X\right\} .
$$

[1] D. Allcock, Braid pictures for Artin groups, Trans. AMS, 354 (2002) 3455-3474.

[2] J. S. Birman, H. Wenzl, Braids, Link polynomials and a new algebra, Trans. AMS, 313 (1989) 249-273.

[3] R. Brauer, On algebras which are connected with the semisimple continuous groups, Annals of Math., 38 (1937) 857-872. 
[4] A. M. Cohen, B. Frenk, and D. B. Wales, Brauer Algebras of simply laced type, preprint, Eindhoven, 2007.

[5] A. M. Cohen, D. A. H. Gijsbers, and D. B. Wales, BMW Algebras of simply laced type, J. Algebra 286 (2005) 107-153.

[6] A. M. Cohen, D. A. H. Gijsbers, and D. B. Wales, The BMW Algebras of Type $\mathrm{D}_{n}$, preprint, Eindhoven, 2007.

[7] D. A. H. Gijsbers, BMW algebras of simply laced type, Ph. D. thesis, Technische Universiteit Eindhoven (2005).

[8] F. M. Goodman, H. Hauschild, Affine Birman-Wenzl-Murakami algebras and tangles in the solid torus, Fundamenta Mathematicae, 190 (2006) 77-137.

[9] R. M. Green, Generalized Temperley-Lieb algebras and decorated tangles, J. Knot Theory and Ramifications 7 (1998), no. 2, 155-171.

[10] R. Häring-Oldenburg, The reduced Birman-Wenzl algebra of Coxeter type B, J. Algebra 213 (1999) 437-466.

[11] H. R. Morton, A. J. Wasserman, A basis for the Birman-Wenzl Algebra, preprint, 1989, liv.ac.uk/ su14/papers/WM.ps.gz.

[12] J. Murakami, The Kauffman polynomial of links and representation theory, Osaka J. Math., 24 (1987) $745-758$.

[13] H. Wenzl, Quantum groups and subfactors of type B, C, and D, Commun. Math. Phys., 133 (1990) 383-432.

Arjeh M. Cohen, Department of Mathematics and Computer Science, Eindhoven University of Technology, POBox 513, 5600 MB Eindhoven, The Netherlands

E-mail address: A.M.Cohen@tue.nl

Dié A.H. GiJsbers,

E-mail address: dahgijsbers@gmail.com

David B. Wales, Mathematics Department, Sloan lab, Caltech, Pasadena, CA 91125, USA

E-mail address: dbw@its.caltech.edu 